\title{
Halogen bonding: an underestimated player in membrane-drug interactions
}

\author{
Rafael Santana Nunes, ${ }^{\dagger}+$ Diogo Vila-Viçosa, ${ }^{\dagger}$ and Paulo J. Costa*,† \\ $\dagger$ University of Lisboa, Faculty of Sciences, BioISI - Biosystems $\&$ Integrative Sciences \\ Institute, Campo Grande, C8 bdg, 1749-016 Lisboa, Portugal \\ $\ddagger$ Centro de Química Estrutural, Faculdade de Ciências, Universidade de Lisboa, Campo \\ Grande, 1749-016 Lisboa, Portugal \\ E-mail: pjcosta@fc.ul.pt \\ Phone: +351-21-7500845
}

\begin{abstract}
Halogen bonds (HaBs) are noncovalent interactions where halogen atoms act as electrophilic species interacting with Lewis bases. These interactions are relevant in biochemical systems being increasingly explored in drug discovery, mainly to modulate protein-ligand interactions. In this work, we report evidence for the existence of HaB-mediated halogen-phospholipid recognition phenomena as our molecular dynamics simulations support the existence of favorable interactions between halobenzene derivatives and both phosphate (PO) or ester (CO) oxygen acceptors from model phospholipid bilayers, thus providing insights into the role of HaBs in driving the permeation of halogenated drug-like molecules across biological membranes. This represents a relevant molecular mechanism, previously overlooked, determining the pharmacological activity of halogenated molecules with implications in drug discovery and development, a place where halogenated molecules account for a significant part of the chemical space.
\end{abstract}


Our data also shows that, as the ubiquitous hydrogen bond, HaBs should be accounted for in the development of membrane permeability models.

keywords: halogen bonding, drug-phospholipid interactions, biomolecular recognition, membrane permeation, molecular dynamics simulations

\section{Introduction}

Proteins and nucleic acids are the most common targets of bioactive molecules or drugs towards therapeutic applications. ${ }^{1}$ Despite the development of strategies aiming at membrane receptors ${ }^{2}$ specific targeting of the membrane phospholipids is much less common. However, in the last few years, new therapeutic approaches have been developed in which lipids are specifically targeted, i.e. membrane-lipid therapy. ${ }^{3}$ Indeed, many bioactive compounds and drug(-like) molecules are membrane-active, interacting directly with membrane lipids, modulating its biophysical properties, and eventually triggering subsequent downstream events which may lead to promiscuous cellular alterations. ${ }^{4-7}$ This interaction is often concomitant with specific therapeutical effects ${ }^{3}$ such as in the case of antimicrobial peptides, ${ }^{8-10}$ sugarbased bactericides ${ }^{11}$ and other small molecule antibiotics, ${ }^{12}$ or modulators of multidrug resistance of tumoral cells. ${ }^{13}$ Therefore, understanding lipid-drug interactions is paramount not only for designing the above-mentioned lipid therapies, ${ }^{3}$ but also to describe phenomena such as membrane permeability, which plays a key role in drug design and development. ${ }^{14-18}$

The interaction and/or diffusion of a drug through a membrane is dependent on a variety of factors, among them, its ability to establish noncovalent interactions. In this scope, the formation of intra- or intermolecular hydrogen bonds (HBs) is a well known crucial factor

affecting membrane permeability and hence determining pharmacological activity. ${ }^{19-23}$ In contrast, the role of other noncovalent interactions, in particular, halogen bonds $(\mathrm{HaBs}),{ }^{24}$ remains unaddressed in the field of membrane-drug recognition.

HaBs consist of $\mathrm{R}-\mathrm{X} \cdots \mathrm{B}(\mathrm{X}=\mathrm{Cl}$, $\mathrm{Br}$, or $\mathrm{I}$; $\mathrm{B}=$ Lewis base; $\mathrm{R}=$ substituent $)$ noncova- 
lent interactions that are predominantly explained by the existence of a localized region of depleted electron density at the tip of the covalently bound halogen atom named $\sigma$-hole. ${ }^{25}$ HaBs have found application in many fields across the chemical and material sciences ${ }^{26,27}$ and, also, in biology. Indeed, since the seminal work of P. Shing Ho and co-workers, ${ }^{28}$ they have been recognized as important players in biomolecular recognition phenomena in protein $^{29,30}$ or nucleic acid ${ }^{31-35}$ systems, and, consequently, have been successfully employed as tools in medicinal chemistry. ${ }^{36-38}$ However, to the best of our knowledge, no reports exist on specific HaB-mediated drug-membrane recognition phenomena. This is surprising since halogen atoms, present in c.a. $25 \%$ of marketed drugs and being even more prevalent in earlier stages of drug discovery and development processes ${ }^{39}$ have traditionally been employed in rational drug design to improve the pharmacokinetic profile of lead molecules, namely to enhance passive diffusion across membranes ${ }^{40}$ or, as recently shown, to increase the receptor-mediated uptake of small fluorescent molecules ${ }^{41,42}$ or proteins ${ }^{43}$ by mammalian cells. ${ }^{44}$ Also, iodination of polymers increases their cellular uptake by plant cells, an effect attributed to HaBs. ${ }^{45}$ None of the reports, however, specifically addressed HaB-mediated passive diffusion.

Several indirect pieces of evidence are pointing out to the potential existence of HaBmediated drug-lipid interactions. HaBs are observed in solution, ${ }^{46}$ including in aqueous media, ${ }^{47,48}$ and can be exploited in the design of synthetic transmembrane anion transporters with potential therapeutic applications. This concept, initially reported by Matile and coworkers ${ }^{49}$ was further developed ${ }^{50-52}$ exploring also other noncovalent interactions. ${ }^{53,54}$ As for anion transporters that are known to interact directly with lipid membranes by establishing HBs with several nucleophilic sites from the phospholipid headgroups, i.e. oxygen atoms, ${ }^{55-57}$ halogen-bonding anion transporters, or any other HaB donor molecule, may eventually engage in an interaction with those sites as well (Figure 1a). Those sites, divided into two dynamic outer membrane regions with the phosphate oxygen (PO) acceptors more exposed to water and the ester oxygen $(\mathrm{CO})$ acceptors located closer to the membrane 
a)
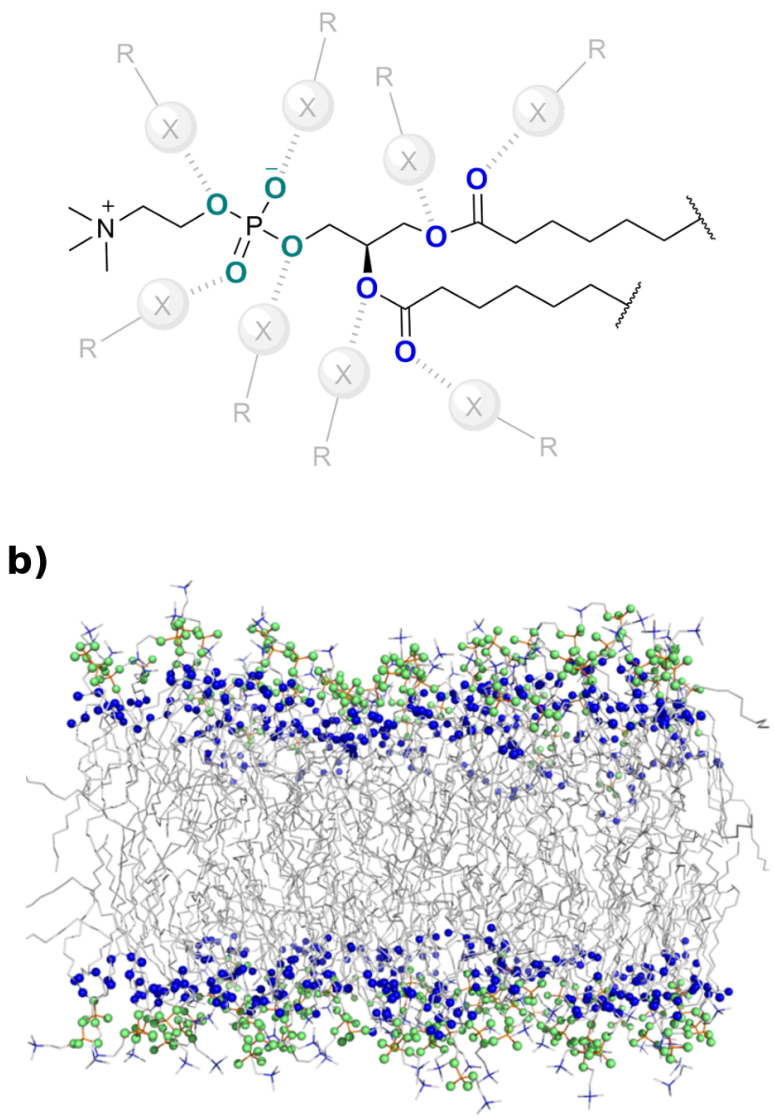

c)
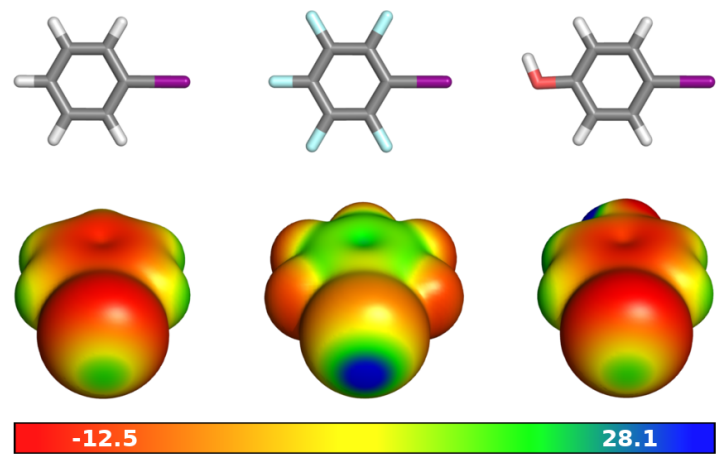

28.1

$V_{\max }=16.9$

$V_{\max }=\mathbf{3 2 . 3}$

$V_{\text {max }}=\mathbf{1 6 . 0}$

Figure 1: (a) Representation of a phosphatidylcholine (PC) molecule highlighting the different lipid HaB- (or HB-) acceptor sites $(\mathrm{X}=\mathrm{Cl}$, Br, I or $\mathrm{OH}$; $\mathrm{R}=$ substituent); (b) PO(green) and CO-type (blue) acceptor layers in a representative POPC bilayer; (c) Iodobenzene derivatives studied in this work, their molecular electrostatic potentials (ESPs) mapped on the 0.001 au contour of the electron density (values in $\mathrm{kcal} \mathrm{mol}^{-1}$ ), and $V_{\max }$ at I (and $\mathrm{OH}$ for iphen) computed at the B3LYP/6-311G(d,p) level. 
core (Figure 1b, green and blue, respectively), bear resemblances with previously reported $\mathrm{HaB}$ acceptors. Indeed, concerning CO-type acceptors, carbonyl oxygens from the protein backbone are frequent and easily targeted $\mathrm{HaB}$ acceptors, ${ }^{28,58,59}$ while in the case of PO interaction sites, the oxygen atoms of phosphines and phosphates are known HaB acceptors, ${ }^{60-64}$ the most remarkable case being the phosphate oxygens from nucleic acids. ${ }^{28,31,35}$ In addition, it was recently shown that both $\mathrm{HB}$ and $\mathrm{HaB}$ interactions participate in the stabilization of complexes between halothane, a well known volatile anesthetic agent, and a lipid phosphate acceptor model (hexamethylphosphortriamide), both in the solid-state and in solution. ${ }^{64}$ This study supports the concept that HaBs might play a role, similarly to HBs, in the molecular recognition of drugs and other small molecules by phospholipids from cell membranes.

Despite all the above-mentioned data suggesting the occurrence of small molecule-membrane $\mathrm{HaB}$ interactions, no direct evidence, similar to that gathered for proteins and nucleic acids $^{28-30,35,65}$ has been reported yet. The identification of the targets of small molecules by experimental techniques, although crucial, is not an easy task ${ }^{66}$ and, in this particular case, identifying an $\mathrm{HaB}$ in a fluid membrane environment might be challenging to achieve experimentally by standard techniques. The existence of such interaction might open an avenue for future drug design, specifically when lipids are directly targeted, ${ }^{3}$ and in this context, in silico methods may provide useful insights into the study of such events at the molecular level.

Herein, we present the first account addressing halogen bonding in membrane-drug interactions and their potential role in mediating passive membrane permeability / internalization at the molecular level. This was accomplished using molecular mechanics / molecular dynamics (MM/MD) simulations, explicitly accounting for the $\sigma$-hole in halogenated species (see Methods), using a model POPC bilayer, together with a series of iodobenzene derivatives (Figure 1c) that are commonly used as probes to evaluate the HaB-capability of small molecules in MD simulations of protein-ligand systems. ${ }^{67-69}$ Given the significant part of 
the chemical space occupied by halogenated molecules in the framework of drug discovery and development, we hope to provide new tools for rational drug design, also contributing to improve the commonly used molecular descriptors for membrane permeation models.

\section{Methods}

\section{Model Systems}

To study the potential role of HaBs in membrane-drug recognition phenomena, the cell membrane was modeled as a 1-palmitoyl-2-oleoyl-sn-glycero-3-phosphocholine (POPC) bilayer. ${ }^{55-57}$ A series of iodobenzene derivatives (Figure 1c), capable of establishing HaBs, were chosen as probes. Iodopentafluorobenzene ( $5 \mathrm{fibz}$ ), exhibiting a considerable $\sigma$-hole corresponding to a local maximum on the electrostatic potential $\left(V_{\max }\right)$ of $32.3 \mathrm{kcal} \mathrm{mol}^{-1}$ (B3LYP/6-311G(d,p)) and a HaB-mediated transmembrane anion transport activity $\left(\mathrm{EC}_{50}\right)$ of $260 \mu \mathrm{M}^{49}$ was selected as a strong $\mathrm{HaB}$ donor probe together with iodobenzene (ibz) which is a considerably weaker $\mathrm{HaB}$ donor $\left(V_{\max }=16.9 \mathrm{kcal} \mathrm{mol}^{-1}\right)$ and exhibits much negligible transmembrane anion transport activity $\left(\mathrm{EC}_{50}>2 \mathrm{mM}\right) .{ }^{49} \mathrm{In}$ addition, 4-iodophenol (iphen), which is also a weak HaB donor $\left(V_{\max }=16.0 \mathrm{kcal} \mathrm{mol}^{-1}\right)$, was selected as a model halogen- and hydrogen-bonding probe to inspect the competing effect of the two intermolecular interactions. Notice that HaBs and HBs have a complex relationship, either competing, replacing, or behaving independently from each other. ${ }^{27,70}$

\section{Probe Parametrization}

To account for HaBs in MM/MD simulations, the halobenzene derivatives were parameterized employing an extra-point $(\mathrm{EP})$ model $^{71-74}$ implemented in the context of the general AMBER force field (GAFF). ${ }^{75}$ In this approach, the halogen atom is modeled by introducing a positively charged particle (EP) mimicking the $\sigma$-hole, thus emulating the charge anisotropy. This strategy was successfully applied in a variety of computational studies 
of HaB-mediated biomolecular recognition using MD simulations, ${ }^{76-79}$ including variants of the original parametrization. ${ }^{67,68,80,81}$ The EP is commonly located at a distance from the halogen corresponding to its Lennard-Jones (LJ) parameter $R_{\min }$ (i.e. $d_{\mathrm{X}-\mathrm{EP}}=R_{\min }$ ) and atomic partial charges are subsequently derived for all atoms by a restrained electrostatic potential (RESP) ${ }^{82}$ fitting procedure, although other authors have proposed alternative EP parametrization schemes in the same context. ${ }^{83-87}$ The EP approach is easily ported to other force fields, ${ }^{38}$ namely OPLS, ${ }^{88-90} \mathrm{CHARMM}^{91}$ or GROMOS,${ }^{69}$ and is compatible with Poisson-Boltzmann and surface area (PBSA) $)^{92,93}$ or generalized Born (GBSA) ${ }^{94,95}$ calculations for estimating protein-ligand binding free energy or hydration free energies. Notice that, despite other less standard approaches being available ${ }^{38,96}$ including force fields specifically designed for biological applications, ${ }^{97,98}$ based on QM data, ${ }^{99}$ or featuring explicit terms to account for polarization effects, ${ }^{100-102}$ these however are not easily generalized for standard force fields.

Accordingly, the molecular electrostatic potential (ESP) at the HF/6-31G(d) ${ }^{103-105}$ level of theory (6-311G(d) ${ }^{106}$ basis set in the case of iodine) was generated for the three iodinated molecules, previously optimized at the B3LYP/6-311G(d,p) level of theory, using Gaussian $09 .{ }^{107}$ The atomic radius of iodine was set to $2.3 \AA,{ }^{108}$ similarly to previous work, ${ }^{69,93}$ while default Merz-Singh-Kollman (MK) radii were employed for the remaining elements. The calculations were performed using four concentric layers of points per atom and six points per unit area with the input options IOp $(6 / 33=2,6 / 41=4,6 / 42=6)$. An EP was then introduced along the $\mathrm{C}-\mathrm{I}$ covalent bond axis, with the $\mathrm{C}-\mathrm{I}-\mathrm{EP}$ angle fixed at $180.0^{\circ}$ and a I-EP distance of $2.15 \AA$, which corresponds to the $R_{\min }$ value for iodine in current versions of GAFF, as previously noted. ${ }^{38,93}$ Atomic partial charges were subsequently derived by RESP, which was carried out using the antechamber ${ }^{109}$ module as implemented in AmberTools $15,{ }^{110}$ thus generating models $i b z^{\mathrm{EP}}, 5 f i b z^{\mathrm{EP}}$, and $i p h e n^{\mathrm{EP}}$. Probe topologies were generated by assigning GAFF atom types with the leap tool (Ambertools 15), and converted into GROMACS-compatible format using the acpype ${ }^{111}$ tool. The EPs were 
modeled as GROMACS type 2 virtual sites defined by the respective $\mathrm{C}-\mathrm{I}$ bond, and without additional parameters, similar to previous work. ${ }^{69}$ Probe models without EP $\left(i b z^{\text {noEP }}\right.$, $5 f i b z^{\text {noEP }}$, $\left.i p h e n^{\text {noEP }}\right)$ were also parameterized following a similar strategy. The full sets of charges (Figure S1a) and final topology files (.itp format) are provided as Supporting Information.

\section{System Setup}

The systems were built from a pre-equilibrated POPC bilayer containing 128 lipids solvated with 5652 water molecules. As mentioned above, ibz, 5 fibz and iphen were selected as probes to assess different halogen-phospholipid interactions and their relative preferences. Individual simulations of each system were performed by adding two probe molecules either into the interior of the lipid bilayer or in the water phase (Figure S2). Several simulation

replicates were run for each setup. For all systems and replicates, we observed that, in the first scenario, the halobenzenes remained inserted in the lipid phase throughout all the simulation time while, in the second case, the molecules inserted into the bilayer at different time scales, remaining inserted during the remainder simulation time (Figures S3-S8). After insertion, the simulations are in equilibrium and both scenarios are indistinguishable. The first $45 \mathrm{~ns}$ of the simulation (when starting from the membrane core) or the time prior to insertion (starting from the water phase) were discarded as equilibration time. Since the two probe molecules do not aggregate and typically do not interact with each other, each corresponding trajectory was also treated separately as individual replicates for analysis purposes. Overall, statistics was performed over 10 individual replicates of $160 \mathrm{~ns}$ each, yielding a total of $1.6 \mu \mathrm{s}$ of sampling time for each system. The time evolution of all replicates and the 160 ns segments used for analysis are highlighted in Figures S3-S8. Since we were also interested in the potential HaB-mediated insertion process, the non-equilibrium segments of the previously mentioned simulations (with the probes starting in the water phase and subsequently inserting in the membrane) were also analyzed. For this purpose, additional 
simulations starting from the water phase were performed until 10 replicates of individual insertion events were sampled for each halobenzene-POPC system.

\section{MM/MD Settings}

Molecular mechanics/molecular dynamics (MM/MD) simulations were performed using the GROMACS software package, versions 5.1.2 and 5.1.5. ${ }^{112-114}$ The AMBER lipid FF (Lipid14 release) ${ }^{115}$ was used for POPC together with TIP3P ${ }^{116}$ for water, as described in ref. 115. The halobenzene molecules were modeled in the framework of GAFF ${ }^{75}$ (with or without an EP), as described above. A tetragonal simulation box was employed, using three-dimensional periodic boundary conditions with the minimum image convention. The simulations were performed in an isothermal-isobaric (NPT) ensemble, with the temperature maintained at $303 \mathrm{~K}$ using the velocity-rescale algorithm ${ }^{117}$ and a coupling constant of $0.1 \mathrm{ps,}$ while pressure was kept constant at 1 bar using a semi-isotropic Berendsen barostat ${ }^{118}$ with a coupling constant of $1.0 \mathrm{ps}$ and an isothermal compressibility of $4.5 \times 10^{-5} \mathrm{bar}^{-1}$. Electrostatic interactions were treated using the smooth particle mesh Ewald (PME) method 119,120 with a Fourier grid spacing of $0.16 \mathrm{~nm}$ and a real-space cutoff of $1.0 \mathrm{~nm}$. Lennard-Jones interactions were truncated at $1.0 \mathrm{~nm}$. The buffered Verlet list scheme ${ }^{121}$ was used for neighbor searching.

The parallel version of the linear constraint solver (P-LINCS) ${ }^{122,123}$ algorithm was used to constrain all lipid and probe bonds, while the SETTLE ${ }^{124}$ algorithm was used for water. Energy minimization was performed in two steps using the steepest descent algorithm until reaching machine precision, in a first step without constrains, followed by a final step with all bond lengths constrained. All simulations were initialized in three steps: (i) initially, random velocities were generated from a Maxwell-Boltzmann distribution at $303 \mathrm{~K}$ and a 100 ps simulation was performed with the positions of all lipid and probe atoms restrained using a force constant of $1000 \mathrm{~kJ} \mathrm{~nm}^{-2} \mathrm{~mol}^{-1}$, followed by (ii) further $100 \mathrm{ps}$ with only the probe atoms restrained $\left(1000 \mathrm{~kJ} \mathrm{~nm}^{-2} \mathrm{~mol}^{-1}\right)$ and (iii) a final $50 \mathrm{ps}$ unrestrained simulation. 
The equations of motion were integrated with the leapfrog algorithm using a time step of 2 fs, with conformations being saved every 5 steps.

\section{Analysis}

The lipid-probe intermolecular interactions (HaBs or HBs) were analyzed using a criterion based on the interaction potential energy ( $E_{\text {pot }}$, see Results and Discussion). For that purpose, we computed $E_{\text {pot }}$ for the interaction between iodine, the EP (when applicable),

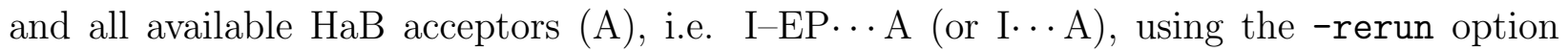
of the gmx mdrun GROMACS tool. All 8 headgroup oxygen atoms (see Figures 1a and S1b) from the 128 phospholipid molecules in the system were considered as potential and independent $\mathrm{HaB}$ acceptors. For each frame, only the lowest $E_{\text {pot }}$ was considered for $\mathrm{HaB}$ evaluation. A similar methodology was used to analyze HB interactions with iphen, in this case, by computing $E_{\text {pot }}$ values for all potential O-H $\cdots$ A interactions instead. For comparison purposes, intermolecular interactions were alternatively analyzed based on geometrical properties, i.e. using the I $\cdots$ A distance and the $\mathrm{C}-\mathrm{I} \cdots \mathrm{A}$ angle for the 10 closest interactions as descriptors. ${ }^{69}$

The probability density functions were estimated using a Gaussian kernel, ${ }^{125}$ and the resulting probability densities were converted into free energies:

$$
E(\mathbf{r})=-R T \ln \frac{P(\mathbf{r})}{P_{\max }}
$$

where $\mathbf{r}$ is the coordinate along the $1 \mathrm{D}$ or $2 \mathrm{D}$ space and $P_{\max }$ is the maximum value of the probability density function, $P(\mathbf{r}) .{ }^{126}$ Free energy landscapes were used to represent the 2D space generated from the simulation data by computing pairs of $E_{\text {pot }}$ (or I ‥ A distance, C-I - . A angle) coordinates.

The probe distribution across the bilayer normal, or membrane insertion along the simulation time, was determined as the $z$ distance between iodine (or oxygen in the case of $i p h e n$ ), 
or the center-of-mass (COM) of the probe and the average $z$-position of all phosphorus atoms in the nearest leaflet.

All reported error bars correspond to the standard error of the mean between the 10 independent replicates. Rendered structures were obtained with PyMOL. ${ }^{127}$

\section{Results and Discussion}

The potential role of HaBs in drug-lipid interactions was investigated using biomolecular simulations. The configurational space was analyzed with respect to the relative $\mathrm{HaB}$ sampling involving different acceptor types (CO and PO) from lipid headgroups, differing in their positioning across the bilayer normal and their relative strength as potential HaBacceptors (Figure 1a,b), and iodobenzene derivatives with distinct HaB-donor capabilities (ibz, $5 \mathrm{fibz}$ and iphen, Figure 1c). In the case of systems featuring iphen, the presence of competing interactions, i.e. hydrogen bonds (HBs), was also evaluated. In addition, the non-equilibrium insertion events sampled in the simulations were also analyzed, providing evidence for a ubiquitous role for $\mathrm{HaB}$ interactions in (halo)molecule permeation across biological membranes. Also, for these studies, establishing robust criteria for HaB assignment is paramount and will be our first step.

\section{HaB Sampling and Assignment Criteria}

$\mathrm{HaB}$ interactions are often evaluated using geometrical criteria, ${ }^{69}$ i.e. a $\mathrm{C}-\mathrm{X} \cdots \mathrm{A}$ angle larger than $140^{\circ}$ and a X . . A distance shorter than the sum of the respective van der Waals radii, in agreement with the values typically employed in crystallographic studies or database surveys. ${ }^{39,59,128,129}$ This type of approach, however, has a few shortcomings. By representing the configurational space as a free energy surface using I $\cdots$ A distances and C-I $\cdots$ A angles for a given acceptor type (CO-type oxygen acceptors for $i b z^{\mathrm{EP}}$ simulations in Figure 2a; Figure S9 for the remainder systems and acceptors), a local free energy minimum is ob- 
served at the typical $\mathrm{HaB}$ region (i.e. $\mathrm{I} \cdots \mathrm{O}<3.5 \AA$ and $\mathrm{C}-\mathrm{I} \cdots \mathrm{O}>140^{\circ}$ ). However, the conformations enclosed within this region (Figure 2b) include many high-energy interactions featuring large deviations from linearity, leading to false-positive HaB assignments (very distorted conformations), while simultaneously not accounting for more elongated interactions sampled that lead to the local minimum centered at the HaB region. Another evidence for a potential erroneous assignment of HaBs is gathered from the surfaces obtained from simulations without an EP where the HaB region is marginally sampled, without a preferred orientation, leading also to false positives (Figure S10).

To solve this issue, the presence of halobenzene-phospholipid HaB interactions was evaluated using the I-EP...A interaction potential energy $\left(E_{\text {pot }}\right)$, resembling the approach used for HB assignment in protein secondary structures. ${ }^{130}$ Indeed, all the potential energy surfaces (PES) computed for the interactions involving the different lipid oxygen acceptors (Figures S11-S13) feature a well-defined energy minimum, centered at the HaB region, showing that $\mathrm{HaB}$ energetics are well described in the simulations in the presence of an EP, whereas without EP addition this is not observed, as expected (Figures S14-S16). Generating the free energy in the $1 \mathrm{D} E_{\text {pot }}$ space, from its probability density, a well-defined maximum value separating a region corresponding to $\mathrm{HaBs}$ from other unspecific contacts (global minima) is obtained (Figures 2c and S17). The $E_{\text {pot }}$ value associated with this free energy maximum can be employed as a cutoff for assigning HaB interactions for each system and acceptor type. Following this approach, the high free-energy interactions that would otherwise be assigned as HaBs according to geometrical criteria (Figure 1d, violet dots) are replaced by the more elongated interactions effectively corresponding to HaBs in the MM/MD simulations as noted above (Figures 1d, yellow dots) that otherwise would be excluded. An illustration of this point is shown in Figure S18. Using the $E_{\text {pot }}$ criterion, the yellow- and green-dotted regions in the free energy landscape are considered as HaBs (Figure 1d). This strategy also eliminates false positives when running simulations without an EP as no free energy minimum corresponding to HaBs exists on the 1D $E_{\text {pot }}$ space. 


\section{a)}

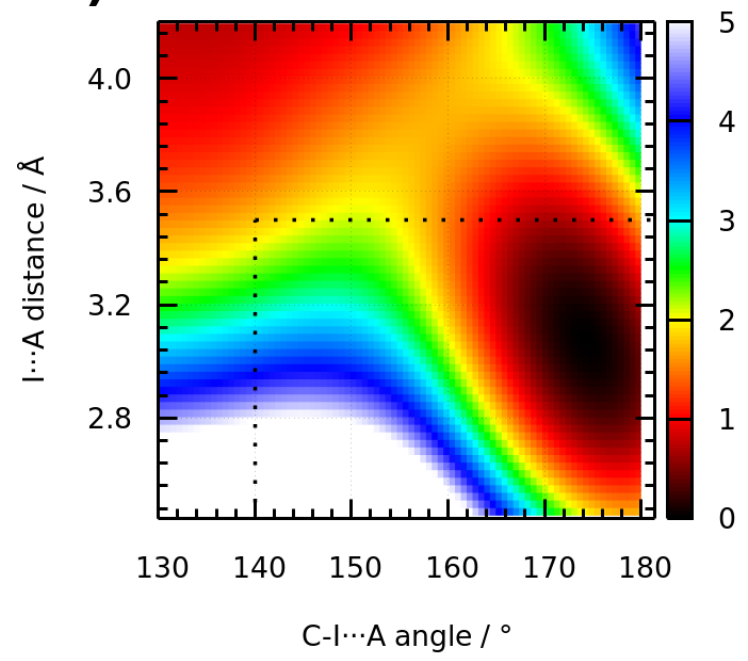

c)

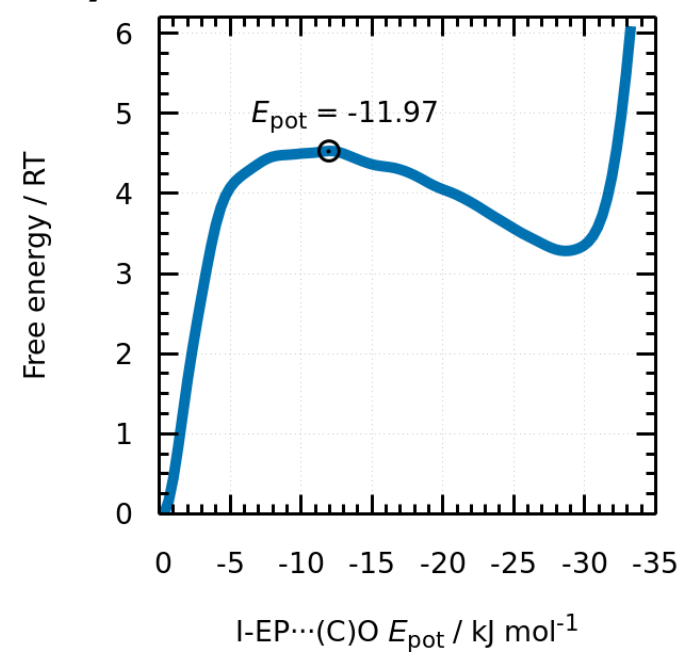

b)
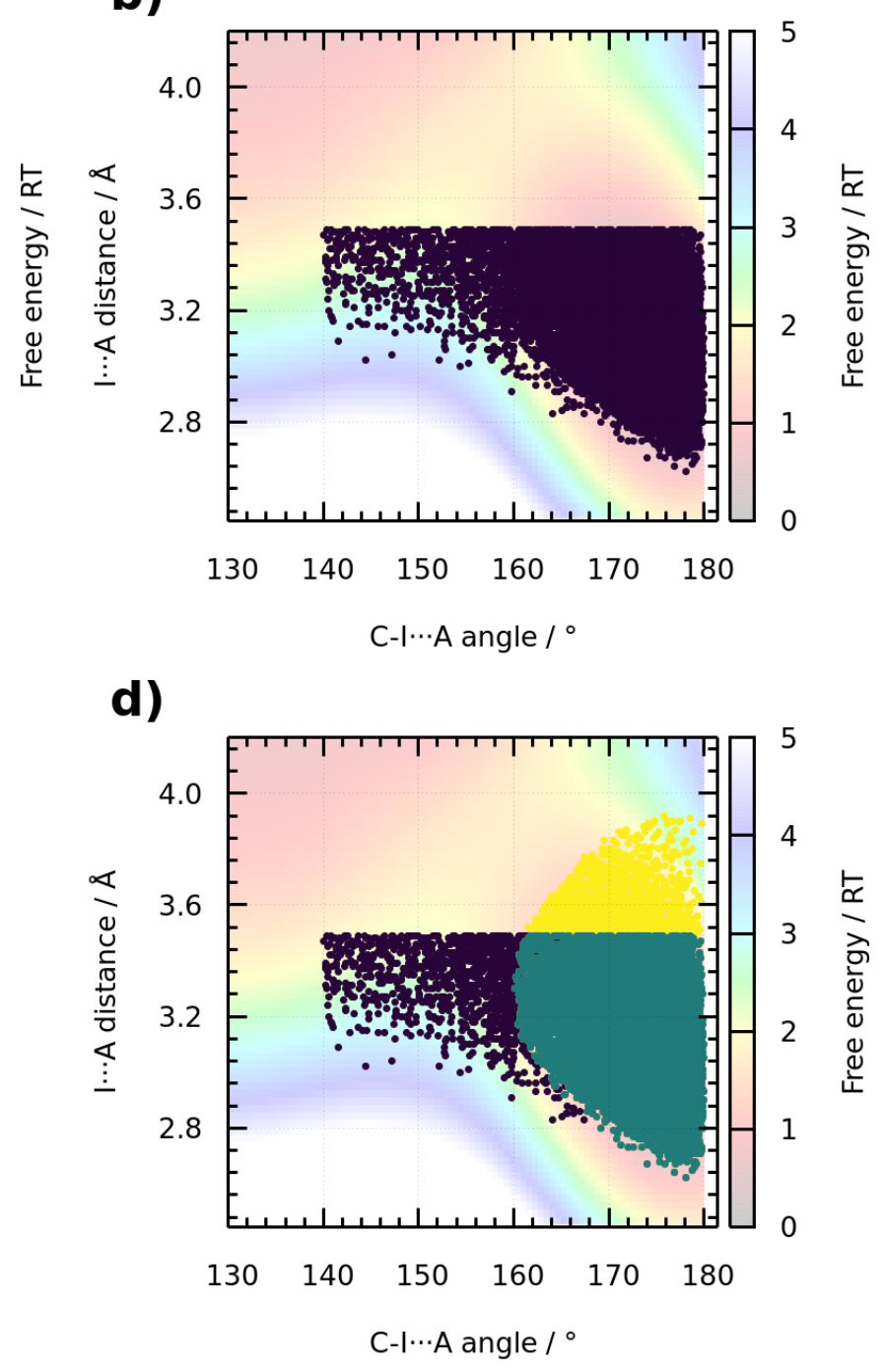

Figure 2: HaB sampling according to geometrical or potential energy $\left(E_{\text {pot }}\right)$ criteria illustrated for $\mathrm{CO}$ acceptors in $i b z^{\mathrm{EP}}$ simulations. (a) Free energy surface using $\mathrm{I} \cdots \mathrm{A}$ distances and C-I . . A angles. The dash-limited region identifies typical HaBs according to geometrical criteria; (b) HaBs assigned by applying the geometrical criteria (in violet); (c) Free energy curve for the distribution of I-EP $\cdots$ A $E_{\text {pot }}$ values for CO-type acceptors highlighting the cutoff value for assigning HaBs; (d) HaBs assigned using the $E_{\text {pot }}$ criterion (in yellow) and overlap with the geometrical criteria (in green). 


\section{HaBs in Membrane-Drug Interactions}

As shown in the previous section, $E_{\mathrm{pot}}$ is a proper criterion to assign HaBs. Hence, the halobenzene-phospholipid $\mathrm{HaB}$ interaction preferences can be evaluated by representing

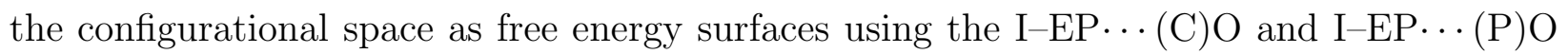
$E_{\text {pot }}$ values (Figures 3 and S19). The free energy landscapes exhibit two local minima at low $E_{\text {pot }}$ values corresponding to $\mathrm{PO}$ - and CO-type $\mathrm{HaB}$ interactions that are interconnected by a global minimum at $E_{\mathrm{pot}}$ around 0, corresponding to other non-specific contacts, thus showing that, in addition to providing a more robust $\mathrm{HaB}$ assignment, this simple description of the system allows also to clearly discriminate between the two types of HaBs. An interesting feature of the landscape is the fact that simultaneous $\mathrm{HaB}$ interactions involving different acceptor types (i.e. "bifurcated" interactions with both PO- and CO-type acceptors) are not sampled in the simulations, even though numerous neighboring acceptors may be available for interacting in a highly dynamic bilayer environment.

When comparing the surface representations obtained for all systems (Figure S19), the $E_{\text {pot }}$ minima corresponding to HaBs follow the order $5 f i b z^{\mathrm{EP}}<i b z^{\mathrm{EP}} \approx i p h e n^{\mathrm{EP}}$, in agreement with the relative $\mathrm{HaB}$ donor strength of the three probes (Figure 1c). Additionally, $\mathrm{HaB}$ interactions involving PO acceptors (1, Figure 3$)$ are associated with lower $E_{\text {pot }}$ values when compared to CO-type interactions (2), as expected given the overall stronger $\mathrm{HaB}$ acceptor character of phosphate oxygens, particularly the more charged $\mathrm{sp}^{2}$-hybridized acceptors (Figures 1a and S1b). In the case of CO-type acceptors, HaBs are mostly formed with the carbonyl oxygens whereas the ester acceptors only account for c.a. $1 \%$.

The probability of I $\cdots$ O interactions effectively corresponding to HaBs was evaluated for each system and acceptor type (Figure 4). The results provide remarkable evidence for the formation of membrane-halobenzene HaBs. The probability can reach a value of c.a. 0.23 in the case of $5 f i b z^{\mathrm{EP}}$, thus highlighting its potential importance in mediating membrane-

drug interactions. For all systems, the probability of $\mathrm{HaB}$ formed with phosphate oxygen atoms (PO-type) is significantly lower than the observed for CO-type acceptors, contrasting 

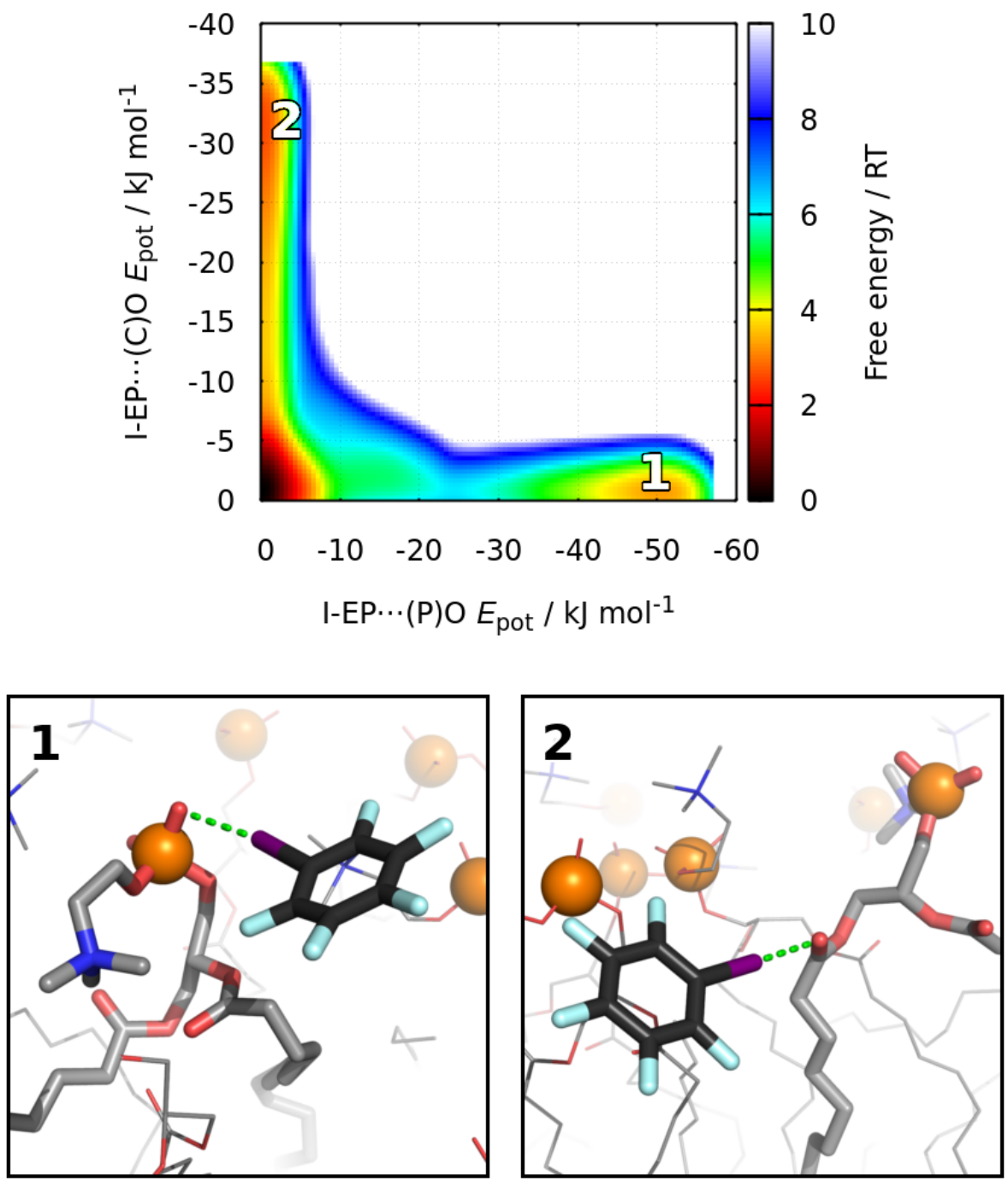

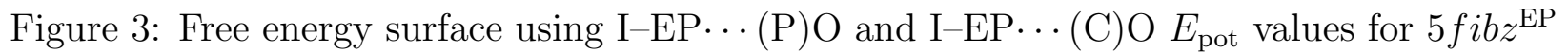
simulations. Representative snapshots are shown for halogen-bonded conformations involving either PO- (1) or CO-type (2) acceptors. $5 f i b z^{\mathrm{EP}}$ and the interacting POPC molecule are shown as sticks with the corresponding $\mathrm{HaB}$ interactions show as green dashes, phosphorus atoms are shown as spheres, non-polar hydrogens and water molecules were omitted for clarity. 


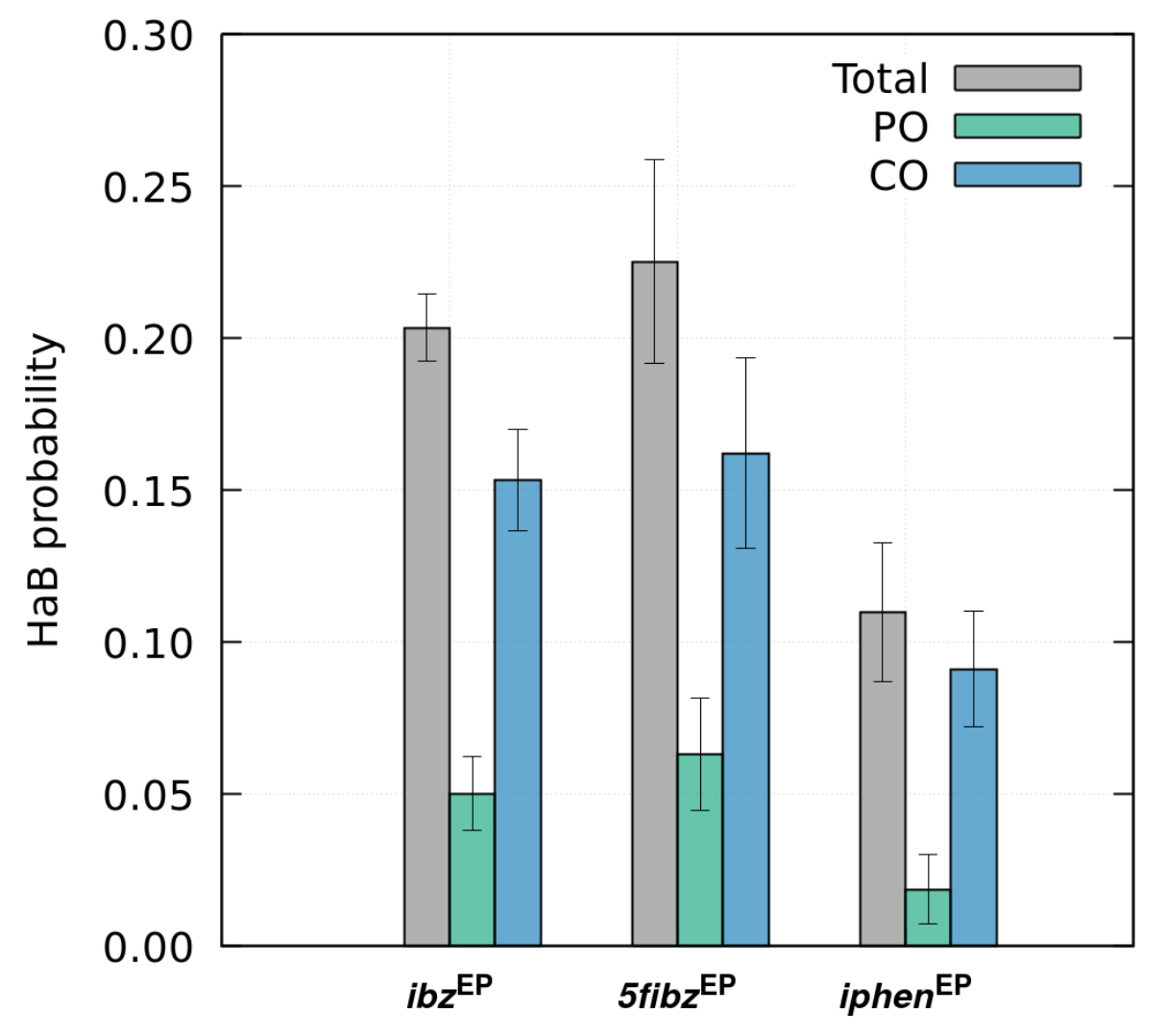

Figure 4: HaB probability for all simulated systems. 
with their relative $E_{\text {pot }}$ values. This is due to the fact that halobenzene molecules, owing to their intrinsically hydrophobic character, populate the inner (ester) region of the membrane more favorably (Figure S20) and, therefore, the potentially stronger phosphate acceptors are less accessible for establishing HaBs. Additionally, the phosphate region is more water exposed whereas the less polar ester region has a stabilizing effect on electrostatically-driven interactions. Note that using these criteria, and with the probes modeled without the EP, no HaBs are assigned in any system.

The total probability of HaBs in $i b z^{\mathrm{EP}}$ simulations (c.a. 0.20 ) is similar to the obtained for $5 f i b z^{\mathrm{EP}}$ (c.a. 0.23), while the number of HaBs is significantly lower for $i p h e n^{\mathrm{EP}}$ (c.a. 0.11). The same trend is observed for the probability of PO- or CO-type HaB interactions. The lower $\mathrm{HaB}$ probability observed for iphen is expected given its mild HaB donor capability and the fact that this molecule is capable of establishing competing HB interactions (see discussion below). In turn, the similar HaB probabilities obtained for $i b z^{\mathrm{EP}}$ and $5 f i b z^{\mathrm{EP}}$ simulations contrast with $5 \mathrm{fibz}$ being a significantly stronger $\mathrm{HaB}$ donor than $i b z$, in spite of a proper description of the donor-strength trend by the respective $E_{\text {pot }}$ minima, as shown above $\left(5 f i b z^{\mathrm{EP}}<i b z^{\mathrm{EP}} \approx i p h e n^{\mathrm{EP}}\right)$. Hence, other properties should be the driving force for this unexpected trend.

To check the halobenzene distribution and orientation along the membrane normal during the simulations, free energy surfaces using the $\mathrm{C}-\mathrm{I}$ angle with the bilayer normal and the iodine insertion along the bilayer were obtained (Figure 5a). Additionally, the probability of finding an $\mathrm{HaB}$ as a function of iodine insertion along the membrane is shown in Figure 5b. The $5 f i b z^{\mathrm{EP}}$ simulations exhibit a single localized free energy minimum at low iodine insertions, centered at c.a. - $0.6 \mathrm{~nm}$ (corresponding to high HaB probabilities) and the $\mathrm{C}-\mathrm{I}$ vector oriented towards the water phase $\left(\sim 30^{\circ}\right)$. In the case of $i b z^{\mathrm{EP}}$, two well-defined preferences are observed, one similar to the observed for $5 f i b z^{\mathrm{EP}}$ but broader and centered at larger angles $\left(\sim 60^{\circ}\right)$, and another at higher iodine insertions and with the $\mathrm{C}-\mathrm{I}$ vector oriented towards the center of the bilayer. At this deeper insertion values, the HaB proba- 

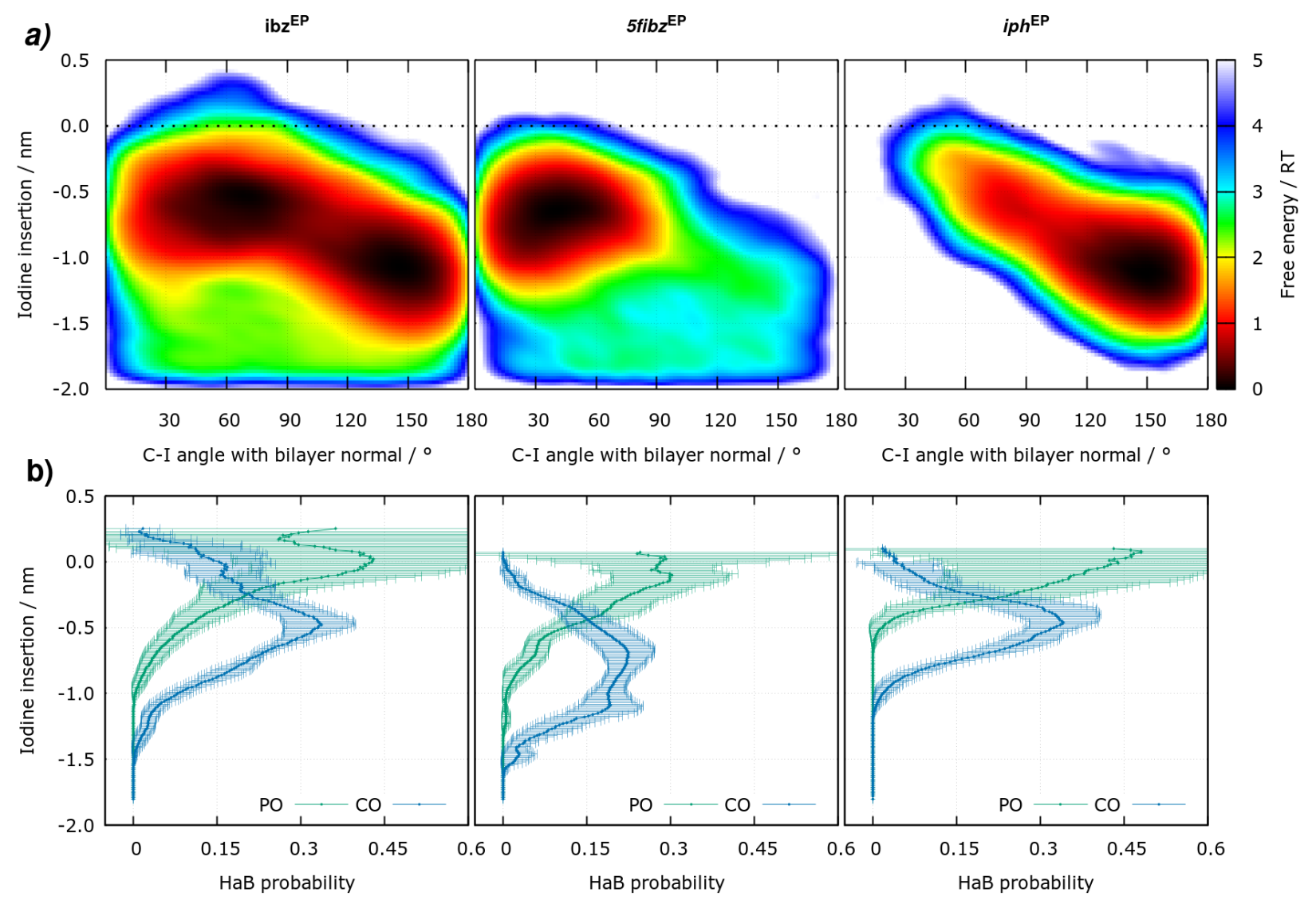

Figure 5: (a) Free energy surfaces using the C-I angle with the bilayer normal and iodine insertion along the bilayer normal. The dashed line corresponds to the average z-position of all phosphorus atoms in the nearest leaflet. (b) HaB probability as a function of iodine insertion along the membrane normal. 
bility is negligible (Figure 5b) as lipid oxygen acceptors are mostly not accessible to engage as interaction partners, and therefore should not account for HaB preferences. However, the former minimum indicates a larger rotational variability when compared with $5 f i b z^{\mathrm{EP}}$, as the molecule may reach $\mathrm{C}-\mathrm{I}$ angles with the bilayer up to $\sim 90^{\circ}$ and consequently establish $\mathrm{HaB}$ interactions, mostly with CO-type acceptors, more favorably. $5 f i b z^{\mathrm{EP}}$, in contrast, is more rotationally restricted, i.e. mostly oriented towards the water phase, therefore having limited access to $\mathrm{HaB}$ acceptors and hence also lower HaB probability (Figure 5b), even though HaBs can occur at higher depths of the membrane $(\geqslant 1 \mathrm{~nm})$, owing to local deformations of the membrane that are driven by the stronger HaB donor character of the molecule. Thus, our results show that despite perfluorination being often used as a strategy to increase membrane permeability, in the case of iodobenzene derivatives, this may lead to a dramatic increase of the $\mathrm{HaB}$ donor properties causing the iodine atom of $5 f i b z^{\mathrm{EP}}$ to populate the inner region of the membrane less favorably (smaller insertions) than $i b z^{\mathrm{EP}}$. Notice, however, that when the center-of-mass (COM) of the molecules are considered, the insertion profiles of the molecules are similar (Figure S20b).

Regarding $i p h e n^{\mathrm{EP}}$, there is a clear preference for conformations featuring the iodine atom inserted closer to the lipid tail region and the $\mathrm{C}-\mathrm{I}$ vector oriented toward the center of the bilayer (Figure 5a), with the $\mathrm{C}-\mathrm{O}(\mathrm{H})$ vector oriented toward the water phase, as expected (see Figure S21a). At the higher values of insertion, the HaB probabilities are negligible (Figure 5b) and the probability of finding an $\mathrm{HaB}$ decreases faster with membrane insertion when compared with $i b z^{\mathrm{EP}}$. Again, this rather restricted rotational freedom, caused by competing HBs (see next section), accounts for a lower HaB probability.

\section{HaBs vs. HBs in Halobenzene-Phospholipid Recognition}

4-Iodophenol exhibits both halogen- and hydrogen-bonding donors, rendering it a suitable model to study the eventual competing effect of the two types of intermolecular interactions that may occur in complex, multi-functionalized drug-like compounds. As previously shown, 
HaBs are less favored for $i p h e n^{\mathrm{EP}}$ (Figure 4), a result that may be driven by the capability of iphen to establish competing HB interactions.

To evaluate this, the same approach developed for HaB was followed, in this case, by computing the $E_{\text {pot }}$ for $\mathrm{O}-\mathrm{H} \cdots(\mathrm{P}) \mathrm{O}$ and $\mathrm{O}-\mathrm{H} \cdots(\mathrm{C}) \mathrm{O}$ interactions. The resulting representation of the configurational space (Figure 6) shows that, similarly to HaBs, the two types of HB interactions involving phosphate- (PO) or ester- $(\mathrm{CO})$ type oxygen acceptors (1 and 2, respectively, in Figure 6) are sampled as free energy local minima being distinguished from other non-specific interactions by this type of analysis. As for HaBs, HB interactions targeting simultaneously the two acceptor types do no occur and, comparing both interactions (Figure 6 and Figure S19, bottom), as expected, HBs are slightly more energetic than HaB interactions. Notice that a very similar free energy surface was obtained for $i p h e n^{\text {noEP }}$ simulations (see Figure S22), showing that EP addition for modeling HaB interactions does interfere with HB sampling.

The relative probability of HBs and HaBs (Figure S23) shows that HBs (c.a. 0.22) are preferred over $\mathrm{HaB}$ interactions (c.a. 0.11) and the same interaction preference for CO-type acceptors is observed for both interactions. However, the probability of HBs targeting POtype acceptors is larger than that found for HaBs, in agreement with the observed preference for deeper iodine insertions (Figure 5a) and more shallow OH insertions (Figure S21a). Also, HB probability is larger at outer regions of the membrane for PO-type acceptors (Figure S21b), however, HBs can still be sampled for CO-type acceptors at inner membrane regions owing to the stronger nature of the interactions which may stabilize the presence of more membrane-inserted lipid headgroups in the simulations.

The eventual occurrence of the two types of interactions (HB and $\mathrm{HaB}$ ) simultaneously was also analyzed allowing to infer if the reported probabilities pertain to exclusively hydrogen- (or halogen-) bonded to conformations or, alternatively, if the two interactions may stabilize iphen-phospholipid binding simultaneously. Indeed, simultaneous HaB and HB interactions are observed, leading to "XHB"-like structures. These configurations correspond, 

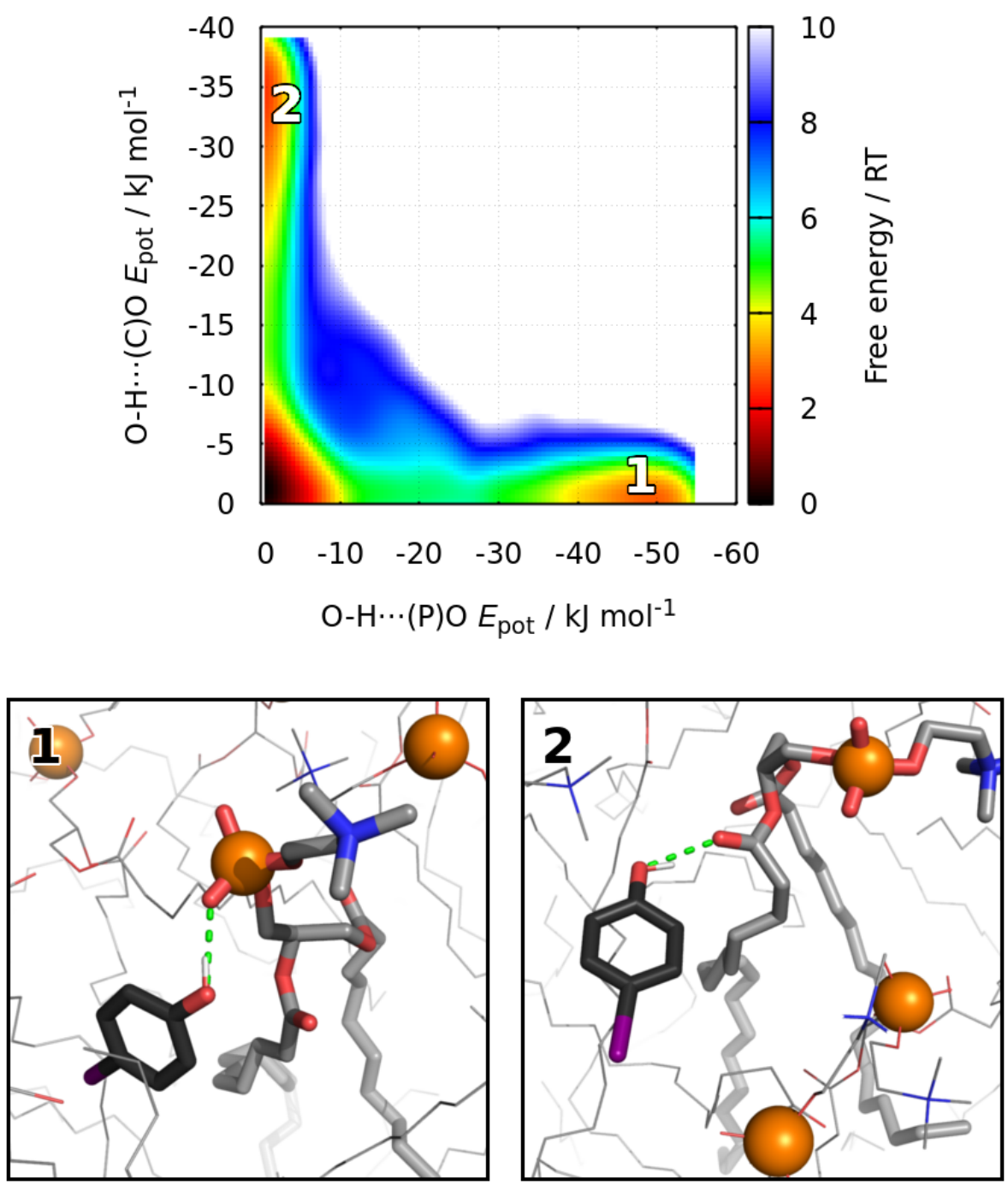

Figure 6: Free energy surface using $\mathrm{O}-\mathrm{H} \cdots(\mathrm{P}) \mathrm{O}$ and $\mathrm{O}-\mathrm{H} \cdots(\mathrm{C}) \mathrm{O} E_{\mathrm{pot}}$ values for $i p h e n^{\mathrm{EP}}$ simulations. Representative snapshots are shown for hydrogen-bonded conformations involving either PO- (1) or CO-type (2) acceptors. HB interactions are shown as green dashes; remainder details as in Figure 3. 


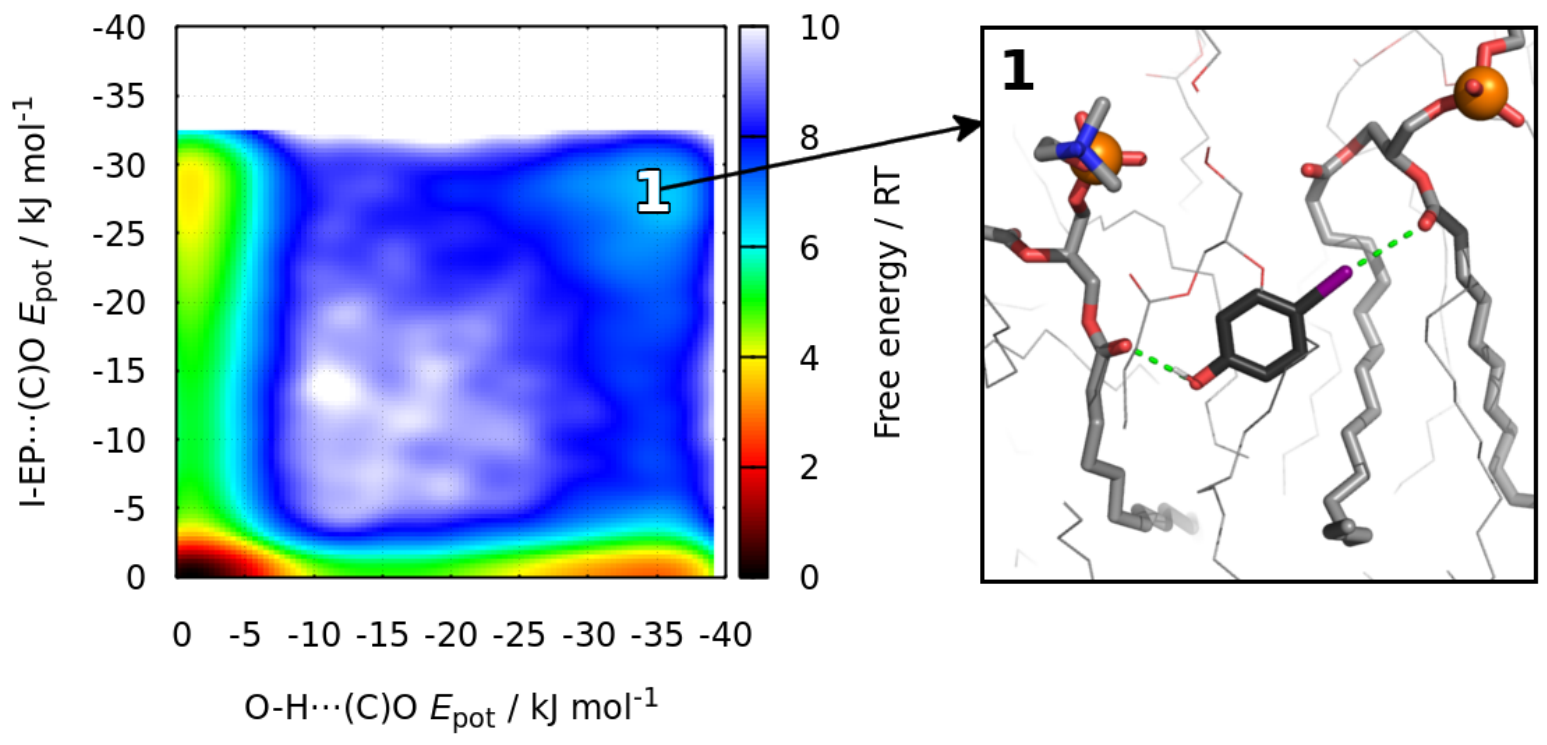

Figure 7: Free energy surface using $\mathrm{O}-\mathrm{H} \cdots(\mathrm{C}) \mathrm{O}$ and I-EP $\cdots(\mathrm{C}) \mathrm{O} E_{\text {pot }}$ values for $i p h e n^{\mathrm{EP}}$ simulations. A representative snapshot for the local minimum corresponding to XHB conformations (accounting for c.a. 1.30\%) is also shown (coloring scheme as in Figure 3).

for example, to two simultaneous $\mathrm{HaB}$ and $\mathrm{HB}$ interactions with ester oxygen (CO)-type acceptors from different phospholipid molecules (1, Figure 7). This type of configuration is the most representative type of XHB interactions observed in the simulations (c.a. 1.30\%). Nonetheless, apart from simultaneous HaBs and HBs with PO-type acceptors, all the other combinations of XHB interactions are observed (Figure S24), including less favorable structures featuring a CO-type $\mathrm{HaB}$ and a PO-type $\mathrm{HB}$ (c.a. 0.66\%), or a PO-type HaB together with a CO-type HB (c.a. 0.49\%), simultaneously (Figure S24). Notice, however, that all types of XHB interactions are scarcely sampled, accounting only for c.a. $2.44 \%$ of all conformations sampled in the simulations (Figure S25). Nevertheless, we show that HaB and HB interactions may not only compete towards the same lipid acceptor but also act cooperatively via two simultaneous bonds with different acceptors in halobenzene-phospholipid recognition phenomena. 


\section{HaB-Mediated Membrane Insertion}

In the previous sections we have shown that, in equilibrium, halobenzene probes interact favorably with phospholipids via HaBs (and HBs) with both phosphate (PO)- and ester (CO)-type lipid oxygen acceptors. In this section, we discuss the role of halogen bonding in the membrane-internalization process by analyzing the non-equilibrium segments of the simulations corresponding to the membrane insertion events sampled in the simulations starting with the ligands in the water phase. The iodine insertion along the membrane normal was monitored while simultaneously evaluating the presence of $\mathrm{HaB}$ (and $\mathrm{HB}$ ) interactions. We were able to distinguish between "on" (interacting) and "off" (non-interacting) states during the insertion process. Figure 8 illustrates the process for representative simulations for all systems (see Figures S26-S28). Halobenzene insertion processes typically occurs in short time-scales $(<6 \mathrm{~ns})$, with the exception of a few replicates, and HaB interactions are frequently sampled preceding the membrane internalization events, i.e. with the halobenzene in the water phase, typically involving the more water-exposed and stronger PO-type acceptors (shown in green) though examples of interactions with CO-type (in blue) acceptors were also identified. Interestingly, $\mathrm{HaB}$ interactions are systematically observed during all insertion events sampled in the simulations, as shown in Figures 8 and S26-S28. These events are characterized by different types of interaction patterns for each replicate / system, though a clear prevalence of a mechanism involving PO-type interaction(s) followed by CO-type interaction(s), as shown in Figure 8, is observed, while other mechanisms, e.g. involving only CO-type interactions, are less common (Figures S26-S28). Furthermore, HaB interactions may either exhibit short kinetics or take place in extended time-scales, depending on the system / replicate. In the case of $i p h e n^{\mathrm{EP}}$ simulations, HB interactions are also frequently established prior to membrane insertion and typically targeting PO-type acceptors (shown in red, Figure S28), however, HaBs are also frequently observed. Indeed, the two types of interactions are systematically observed during the internalization process with their relative interplay determining different interaction patterns (Figure S28). This qualitative analysis 

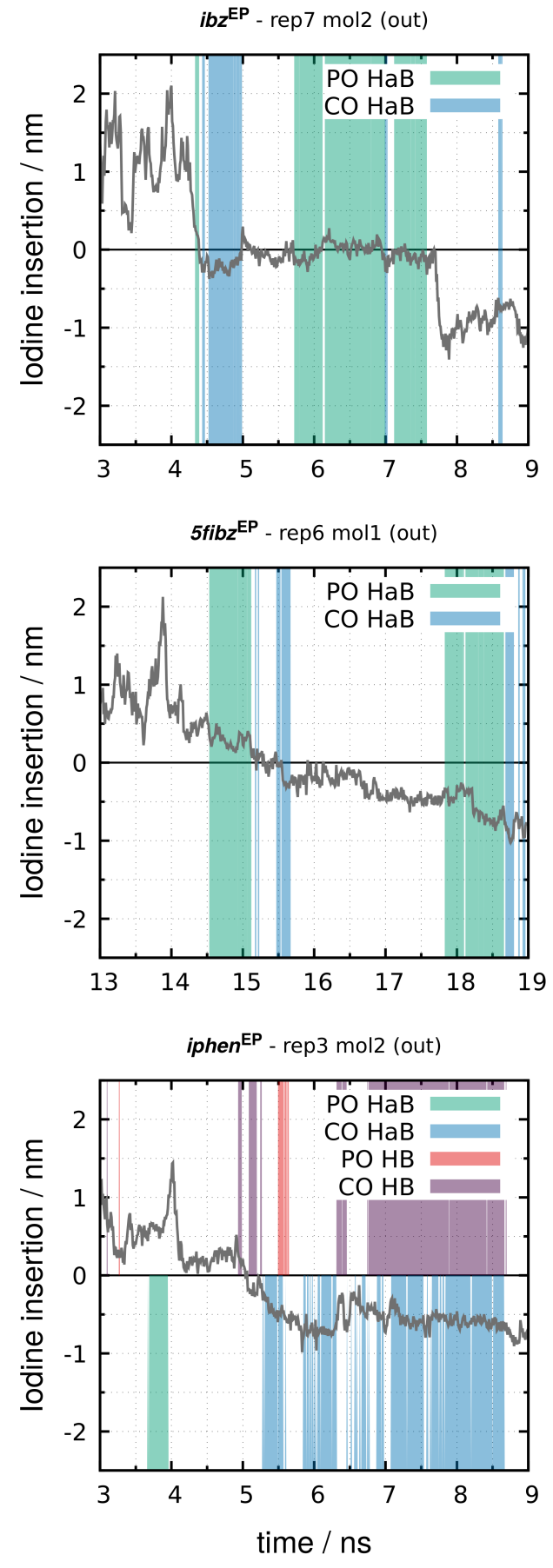

Figure 8: Iodine insertion process for a representative replicate of each system. The solid horizontal line corresponds to the average $z$-position of all phosphorus atoms in the nearest leaflet. The presence of HaBs or HBs ("on" states) is depicted as vertical lines. 
of non-equilibrium insertion events provides evidence supporting the concept that HaB interactions may play a direct role in mediating the permeation of halogenated small molecules across biological membranes, parallel to the well-known ubiquitous role of hydrogen-bonding in HB-donor molecules. ${ }^{19-23}$

\section{Conclusions}

The potential role of halogen bonding in lipidic systems was poorly understood when compared with the recognized importance of $\mathrm{HaB}$ interactions in protein-ligand complexes or nucleic acids. Indeed, to the best of our knowledge, no previous literature reports have addressed HaB-mediated drug-membrane recognition phenomena, despite the well-known prevalence of halogenated molecules in drug discovery and development ${ }^{36-39}$ and the existence of multiple $\mathrm{HaB}$ acceptors in phospholipids, the main constituent of a cell membranes. To explore the existence of halogen-phospholipid interactions, we carried out MM/MD simulations on three model iodobenzene derivatives in a hydrated phospholipid (POPC) bilayer environment, using an extra-point (EP) approach to model the HaB-properties of the halo-

genated species. ${ }^{71-74}$ This methodology, that had been widely applied in the computational investigation of a variety of protein-ligand systems, ${ }^{76-79,92,94,95}$ provided evidences supporting the role of $\mathrm{HaB}$ interactions in drug-lipid recognition events.

$\mathrm{HaB}$ interactions involving both phosphate $(\mathrm{PO})$ and ester $(\mathrm{CO})$ oxygen acceptors were evaluated and assigned from the sampled configurations using a new criterion based on the I-EP. . A ( $\mathrm{A}=$ PO- or CO-type oxygen acceptor $)$ potential energy $\left(E_{\text {pot }}\right)$, providing an accurate description of halogen-bonding in the simulations by removing false positives obtained when using a plain geometrical cutoff. The results show that iodobenzene derivatives interact with model phospholipid membranes via $\mathrm{HaB}$ interactions targeting both PO- and CO-type acceptors, the latter interactions being electrostatically favored due to the less water-exposed environment and intrinsically hydrophobic character of the probe molecules, despite PO-type 
acceptors being stronger $\mathrm{HaB}$ interaction partners. $\mathrm{HaB}$ probability is also dependent on the orientational preferences with respect to the bilayer normal, with perfluorination favoring conformations with the $\mathrm{C}-\mathrm{I}$ vector oriented towards the water phase and hence with a more limited access to $\mathrm{HaB}$ acceptors, compared to the weaker $\mathrm{HaB}$ donor counterparts. On the other hand, the probability to establish HaBs at inner regions of the bilayer effectively increases with the relative $\mathrm{HaB}$ donor strength of the probes, i.e. $5 f i b z^{\mathrm{EP}} \gg i b z^{\mathrm{EP}}>i p h e n^{\mathrm{EP}}$.

Our results also showed that, similarly to other biological systems, ${ }^{27,70}$ HaBs can compete with HBs in the case of small molecules bearing both HaB- and HB-donor moieties such as iphen. Even though $\mathrm{O}-\mathrm{H} \cdots \mathrm{O}$ interactions are slightly stronger than the $\mathrm{I} \cdots \mathrm{O}$ counterparts, the two types of interactions can also occur simultaneously in transient higher free-energy "XHB-like" conformations which may act either cooperatively or competitively in mediating ligand-membrane recognition events.

The non-equilibrium segments of the trajectories corresponding to the membrane insertion events sampled in the simulations starting with the molecules in the water phase were analyzed by monitoring the iodine insertion along the membrane normal over time and simultaneously assessing the presence of $\mathrm{HaB}$ interactions. HaBs were observed in all replicates, often preceding membrane permeation, suggesting a role in directly promoting the passive transport of halogenated drug-like molecules across model biological membranes.

In summary, the role of $\mathrm{HaB}$ interactions in lipid-drug systems was tackled for the first time and our data provide important insights into (halo)drug-membrane recognition mechanisms at the molecular level. In particular, we propose that biomembrane permeation of drug-like compounds can be directly mediated by $\mathrm{HaB}$ interactions implying that, beyond often exhibiting superior pharmacokinetic profile, namely enhanced passive membrane diffusion, ${ }^{40}$ bioactive halogenated molecules may further reach biological targets via direct halogen-phospholipid interactions, which may contribute determinedly to their pharmacological efficacy. This concept opens the possibility for the rational design of novel drugs by taking advantage of halogen-lipid recognition phenomena, and for the improvement of 
molecular descriptors for permeation QSAR models that should account for HaB-capability, similarly to the ubiquitous hydrogen bond, ${ }^{19-23}$ towards novel tools for virtual screening routines.

Finally, we note that while this work reinforces the usefulness of MM/MD simulations in the study HaB-mediated biomembrane recognition phenomena, further investigation of these processes in the context of large datasets of drug-like molecules is envisaged. Moreover, we hope these results will encourage experimental investigation aiming at an improved understanding of halogen-phospholipid interactions at the molecular level.

\section{Supporting Information Available}

Supporting Figures (PDF)

Topology files in ITP format for all of the parameterized halobenzene probes (ZIP)

\section{Acknowledgement}

The authors thank Fundação para a Ciência e a Tecnologia (FCT), Portugal, for the Investigador FCT Program IF/00069/2014, exploratory project IF/00069/2014/CP1216/CT0006 (P.J.C.), doctoral grant SFRH/BD/116614/2016 (R.N.), and strategic project UID/MULTI/04046/2019. This work was financed by Programa Operacional Regional de Lisboa (Lisboa 2020), Portugal 2020, FEDER/FN, and the European Union under project number 28455 (LISBOA-01-0145FEDER-028455, PTDC/QUI-QFI/28455/2017).

\section{References}

(1) Imming, P.; Sinning, C.; Meyer, A. Drugs, their targets and the nature and number of drug targets. Nat. Rev. Drug Discov. 2006, 5, 821. 
(2) Yin, H.; Flynn, A. D. Drugging membrane protein interactions. Annu. Rev. Biomed. Eng. 2016, 18, 51-76.

(3) Escribá, P. V. Membrane-lipid therapy: a new approach in molecular medicine. Trends Mol. Med. 2006, 12, 34-43.

(4) Ingolfsson, H. I. et al. Phytochemicals perturb membranes and promiscuously alter protein function. ACS Chem. Biol. 2014, 9, 1788-1798.

(5) Baell, J. B. Screening-Based Translation of Public Research Encounters Painful Problems. ACS Med. Chem. Lett. 2015, 6, 229-234.

(6) Baell, J. B. Feeling natures PAINS: natural products, natural product drugs, and pan assay interference compounds (PAINS). J. Nat. Prod. 2016, 79, 616-628.

(7) Perkins, R.; Vaida, V. Phenylalanine increases membrane permeability. J. Am. Chem. Soc. 2017, 139, 14388-14391.

(8) Epand, R. M.; Walker, C.; Epand, R. F.; Magarvey, N. A. Molecular mechanisms of membrane targeting antibiotics. BBA Biomembranes 2016, 1858, 980-987.

(9) Li, J.; Koh, J.-J.; Liu, S.; Lakshminarayanan, R.; Verma, C. S.; Beuerman, R. W. Membrane active antimicrobial peptides: translating mechanistic insights to design. Front. Neurosci. 2017, 11, 73.

(10) Hurdle, J. G.; O’neill, A. J.; Chopra, I.; Lee, R. E. Targeting bacterial membrane function: an underexploited mechanism for treating persistent infections. Nat. Rev. Microbiol. 2011, 9, 62-75.

(11) Dias, C. et al. Sugar-based bactericides targeting phosphatidylethanolamine-enriched membranes. Nat. Commun. 2018, 9, 4857.

(12) Dias, C.; Rauter, A. P. Membrane-targeting antibiotics: recent developments outside the peptide space. Future Med. Chem. 2019, 11, 211-228. 
(13) Hendrich, A.; Michalak, K. Lipids as a target for drugs modulating multidrug resistance of cancer cells. Curr. Drug Targets 2003, 4, 23-30.

(14) Lipinski, C. A. Avoiding investment in doomed drugs. Curr. Drug. Discov. 2001, 1, $17-19$.

(15) Matsson, P.; Kihlberg, J. How Big Is Too Big for Cell Permeability? J. Med. Chem. 2017, 60, 1662-1664.

(16) Waring, M. J. Lipophilicity in drug discovery. Exp. Opin. Drug Discov. 2010, 5, 235248.

(17) Yue, Z.; Li, C.; Voth, G. A.; Swanson, J. M. Dynamic Protonation Dramatically Affects the Membrane Permeability of Drug-like Molecules. J. Am. Chem. Soc. 2019, $141,13421-13433$.

(18) Dickson, C. J.; Hornak, V.; Bednarczyk, D.; Duca, J. S. Using membrane partitioning simulations to predict permeability of forty-nine drug-like molecules. J. Chem. Inf. Model. 2019, 59, 236-244.

(19) Lipinski, C. A.; Lombardo, F.; Dominy, B. W.; Feeney, P. J. Experimental and computational approaches to estimate solubility and permeability in drug discovery and development settings. Adv. Drug Deliv. Rev. 2001, 46, 3-26.

(20) Bemporad, D.; Luttmann, C.; Essex, J. Behaviour of small solutes and large drugs in a lipid bilayer from computer simulations. BBA Biomembranes 2005, 1718, 1-21.

(21) Shinoda, W. Permeability across lipid membranes. BBA Biomembranes 2016, 1858, $2254-2265$.

(22) Dickson, C. J.; Hornak, V.; Pearlstein, R. A.; Duca, J. S. Structure-kinetic relationships of passive membrane permeation from multiscale modeling. J. Am. Chem. Soc. 2017, 139, 442-452. 
(23) Rezai, T.; Bock, J. E.; Zhou, M. V.; Kalyanaraman, C.; Lokey, R. S.; Jacobson, M. P. Conformational flexibility, internal hydrogen bonding, and passive membrane permeability: successful in silico prediction of the relative permeabilities of cyclic peptides. J. Am. Chem. Soc. 2006, 128, 14073-14080.

(24) Desiraju, G. R.; Ho, P. S.; Kloo, L.; Legon, A. C.; Marquardt, R.; Metrangolo, P.; Politzer, P.; Resnati, G.; Rissanen, K. Definition of the halogen bond (IUPAC Recommendations 2013). Pure Appl. Chem. 2013, 85, 1711-1713.

(25) Clark, T.; Hennemann, M.; Murray, J. S.; Politzer, P. Halogen bonding: the $\sigma$-hole. J. Mol. Model. 2007, 13, 291-296.

(26) Cavallo, G.; Metrangolo, P.; Milani, R.; Pilati, T.; Priimagi, A.; Resnati, G.; Terraneo, G. The halogen bond. Chem. Rev. 2016, 116, 2478-2601.

(27) Costa, P. J. The halogen bond: nature and applications. Phys. Sci. Rev. 2017, 2, 20170136.

(28) Auffinger, P.; Hays, F. A.; Westhof, E.; Ho, P. S. Halogen bonds in biological molecules. Proc. Natl. Acad. Sci. USA 2004, 101, 16789-16794.

(29) Parisini, E.; Metrangolo, P.; Pilati, T.; Resnati, G.; Terraneo, G. Halogen bonding in halocarbon-protein complexes: a structural survey. Chem. Soc. Rev. 2011, 40, $2267-2278$.

(30) Scholfield, M. R.; Zanden, C. M. V.; Carter, M.; Ho, P. S. Halogen bonding (Xbonding): a biological perspective. Protein Sci. 2013, 22, 139-152.

(31) Voth, A. R.; Hays, F. A.; Ho, P. S. Directing macromolecular conformation through halogen bonds. Proc. Natl. Acad. Sci. USA 2007, 104, 6188-6193.

(32) Parker, A. J.; Stewart, J.; Donald, K. J.; Parish, C. A. Halogen bonding in DNA base pairs. J. Am. Chem. Soc. 2012, 134, 5165-5172. 
(33) Carter, M.; Voth, A. R.; Scholfield, M. R.; Rummel, B.; Sowers, L. C.; Ho, P. S. Enthalpy-entropy compensation in biomolecular halogen bonds measured in DNA junctions. Biochemistry 2013, 52, 4891-4903.

(34) Ford, M. C.; Saxton, M.; Ho, P. S. Sulfur as an acceptor to bromine in biomolecular halogen bonds. J. Phys. Chem. Lett. 2017, 8, 4246-4252.

(35) Koláŕ, M. H.; Tabarrini, O. Halogen Bonding in Nucleic Acid Complexes: Miniperspective. J. Med. Chem. 2017, 60, 8681-8690.

(36) Ho, P. S. Halogen bonding in medicinal chemistry: from observation to prediction. Future Med. Chem. 2017, 9, 637-640.

(37) Wilcken, R.; Zimmermann, M. O.; Lange, A.; Joerger, A. C.; Boeckler, F. M. Principles and applications of halogen bonding in medicinal chemistry and chemical biology. $J$. Med. Chem. 2013, 56, 1363-1388.

(38) Costa, P. J.; Nunes, R.; Vila-Viçosa, D. Halogen bonding in halocarbon-protein complexes and computational tools for rational drug design. Expert Opin. Drug Discov. 2019, 14, 805-820.

(39) Xu, Z.; Yang, Z.; Liu, Y.; Lu, Y.; Chen, K.; Zhu, W. Halogen bond: its role beyond drug-target binding affinity for drug discovery and development. J. Chem. Inf. Model. 2014, 54, 69-78.

(40) Gerebtzoff, G.; Li-Blatter, X.; Fischer, H.; Frentzel, A.; Seelig, A. Halogenation of drugs enhances membrane binding and permeation. ChemBioChem 2004, 5, 676-684.

(41) Ungati, H.; Govindaraj, V.; Mugesh, G. The Remarkable Effect of Halogen Substitution on the Membrane Transport of Fluorescent Molecules in Living Cells. Angew. Chem. Int. Ed. 2018, 57, 8989-8993. 
(42) Ungati, H.; Govindaraj, V.; Nair, C. R.; Mugesh, G. Halogen-Mediated Membrane Transport: An Efficient Strategy for the Enhancement of Cellular Uptake of Synthetic Molecules. Chem. Eur. J. 2019, 25, 3391-3399.

(43) Jakka, S. R.; Govindaraj, V.; Mugesh, G. A Single Atom Change Facilitates the Membrane Transport of Green Fluorescent Proteins in Mammalian Cells. Angew. Chem. Int. Ed. 2019, 58, 7713-7717.

(44) Govindaraj, V.; Ungati, H.; Jakka, S. R.; Bose, S.; Mugesh, G. Directing Traffic: Halogen bond-mediated Membrane Transport. Chem. Eur. J. 2019, 25, 11180-11192.

(45) Wang, T.; Yin, P.; Yang, Y.; Yin, W.; Zhang, S.; Yang, M.; Qin, Y.; Ma, Y.; Lei, Z.; Ma, H. Effect of Element Iodine on the Cell Membrane Transportability of Fluorescent Polymers and Lysosome-Targeted Cell Imaging. ACS Sustainable Chem. Eng. 2019, 7, 6295-6303.

(46) Beale, T. M.; Chudzinski, M. G.; Sarwar, M. G.; Taylor, M. S. Halogen bonding in solution: thermodynamics and applications. Chem. Soc. Rev. 2013, 42, 1667-1680.

(47) Zapata, F.; Caballero, A.; White, N. G.; Claridge, T. D. W.; Costa, P. J.; Félix, V.; Beer, P. D. Fluorescent charge-assisted halogen-bonding macrocyclic halo-imidazolium receptors for anion recognition and sensing in aqueous media. J. Am. Chem. Soc. 2012, 134, 11533-11541.

(48) Langton, M. J.; Robinson, S. W.; Marques, I.; Félix, V.; Beer, P. D. Halogen bonding in water results in enhanced anion recognition in acyclic and rotaxane hosts. Nat. Chem. 2014, 6, 1039-1043.

(49) Jentzsch, A. V.; Emery, D.; Mareda, J.; Nayak, S. K.; Metrangolo, P.; Resnati, G.; Sakai, N.; Matile, S. Transmembrane anion transport mediated by halogen-bond donors. Nat. Commun. 2012, 3, 905. 
(50) Vargas Jentzsch, A.; Matile, S. Transmembrane halogen-bonding cascades. J. Am. Chem. Soc. 2013, 135, 5302-5303.

(51) Jentzsch, A. V.; Matile, S. In Halogen Bonding I: Impact on Materials Chemistry and Life Sciences. Topics in Current Chemistry; Metrangolo, P., Resnati, G., Eds.; Springer International Publishing: Cham, 2014; Vol. 358; pp 205-239.

(52) Ren, C.; Ding, X.; Roy, A.; Shen, J.; Zhou, S.; Chen, F.; Li, S. F. Y.; Ren, H.; Yang, Y. Y.; Zeng, H. A halogen bond-mediated highly active artificial chloride channel with high anticancer activity. Chem. Sci. 2018, 9, 4044-4051.

(53) Vargas Jentzsch, A.; Hennig, A.; Mareda, J.; Matile, S. Synthetic ion transporters that work with anion- $\pi$ interactions, halogen bonds, and anion-macrodipole interactions. Acc. Chem. Res. 2013, 46, 2791-2800.

(54) Benz, S.; Macchione, M.; Verolet, Q.; Mareda, J.; Sakai, N.; Matile, S. Anion transport with chalcogen bonds. J. Am. Chem. Soc. 2016, 138, 9093-9096.

(55) Haynes, C. J.; Moore, S. J.; Hiscock, J. R.; Marques, I.; Costa, P. J.; Félix, V.; Gale, P. A. Tunable transmembrane chloride transport by bis-indolylureas. Chem. Sci. 2012, 3, 1436-1444.

(56) Moore, S. J.; Haynes, C. J.; González, J.; Sutton, J. L.; Brooks, S. J.; Light, M. E.; Herniman, J.; Langley, G. J.; Soto-Cerrato, V.; Pérez-Tomás, R.; Marques, I.; Costa, P. J.; Félix, V.; Gale, P. A. Chloride, carboxylate and carbonate transport by ortho-phenylenediamine-based bisureas. Chem. Sci. 2013, 4, 103-117.

(57) Marques, I.; Colaço, A. R.; Costa, P. J.; Busschaert, N.; Gale, P. A.; Félix, V. Tris-thiourea tripodal-based molecules as chloride transmembrane transporters: insights from molecular dynamics simulations. Soft Matter 2014, 10, 3608-3621. 
(58) Wilcken, R.; Zimmermann, M. O.; Lange, A.; Zahn, S.; Boeckler, F. M. Using halogen bonds to address the protein backbone: a systematic evaluation. J. Comput. Aided Mol. Design 2012, 26, 935-945.

(59) Zhang, Q.; Xu, Z.; Shi, J.; Zhu, W. Underestimated halogen bonds forming with protein backbone in protein data bank. J. Chem. Inf. Model. 2017, 57, 1529-1534.

(60) Chowdhury, B.; Sinha, S.; Ghosh, P. Selective Sensing of Phosphates by a New Bisheteroleptic RuII Complex through Halogen Bonding: A Superior Sensor over Its Hydrogen-Bonding Analogue. Chem. Eur. J. 2016, 22, 18051-18059.

(61) Schaub, T. A.; Sure, R.; Hampel, F.; Grimme, S.; Kivala, M. Quantum chemical dissection of the shortest $\mathrm{P}=\mathrm{O} \cdots \mathrm{I}$ halogen bond: the decisive role of crystal packing effects. Chem. Eur. J. 2017, 23, 5687-5691.

(62) Cabot, R.; Hunter, C. A. Non-covalent interactions between iodo-perfluorocarbons and hydrogen bond acceptors. Chem. Commun. 2009, 2005-2007.

(63) Chudzinski, M. G.; Taylor, M. S. Correlations between computation and experimental thermodynamics of halogen bonding. J. Org. Chem. 2012, 77, 3483-3491.

(64) Nayak, S. K.; Terraneo, G.; Piacevoli, Q.; Bertolotti, F.; Scilabra, P.; Brown, J. T.; Rosokha, S. V.; Resnati, G. Molecular Bases for Anesthetic Agents: Halothane as a Halogen-and Hydrogen-Bond Donor. Angew. Chem. Int. Ed. 2019, 58, 12456-12459.

(65) Ho, P. S. In Halogen Bonding I: Impact on Materials Chemistry and Life Sciences. Topics in Current Chemistry, Vol. 358; Metrangolo, P., Resnati, G., Eds.; Springer International Publishing: Cham, 2014; pp 241-276.

(66) Ziegler, S.; Pries, V.; Hedberg, C.; Waldmann, H. Target identification for small bioactive molecules: finding the needle in the haystack. Angew. Chem. Int. Ed. 2013, 52, $2744-2792$. 
(67) Yang, Y.; Mahmoud, A. H.; Lill, M. A. Modeling of Halogen-Protein Interactions in Co-Solvent Molecular Dynamics Simulations. J. Chem. Inf. Model. 2019, 59, 38-42.

(68) Mahmoud, A. H.; Yang, Y.; Lill, M. A. Improving Atom-Type Diversity and Sampling in Cosolvent Simulations Using $\lambda$-Dynamics. J. Chem. Theory Comput. 2019, 15, $3272-3287$.

(69) Nunes, R.; Vila-Viçosa, D.; Machuqueiro, M.; Costa, P. J. Biomolecular Simulations of Halogen Bonds with a GROMOS Force Field. J. Chem. Theory Comput. 2018, 14, $5383-5392$.

(70) Rowe, R. K.; Ho, P. S. Relationships between hydrogen bonds and halogen bonds in biological systems. Acta Crystallogr. B 2017, B73, 255-264.

(71) Ibrahim, M. A. A. Molecular mechanical study of halogen bonding in drug discovery. J. Comput. Chem. 2011, 32, 2564-2574.

(72) Ibrahim, M. A. A. Performance Assessment of Semiempirical Molecular Orbital Methods in Describing Halogen Bonding: Quantum Mechanical and Quantum Mechanical/Molecular Mechanical-Molecular Dynamics Study. J. Chem. Inf. Model. 2011, 51, 2549-2559.

(73) Ibrahim, M. A. A. AMBER empirical potential describes the geometry and energy of noncovalent halogen interactions better than advanced semiempirical quantum mechanical method PM6-DH2X. J. Phys. Chem. B 2012, 116, 3659-3669.

(74) Ibrahim, M. A. A. Molecular mechanical perspective on halogen bonding. J. Mol. Model. 2012, 18, 4625-4638.

(75) Wang, J.; Wolf, R. M.; Caldwell, J. W.; Kollman, P. A.; Case, D. A. Development and testing of a general amber force field. J. Comput. Chem. 2004, 25, 1157-1174. 
(76) Rosa, M.; Caltabiano, G.; Barreto-Valer, K.; Gonzalez-Nunez, V.; GómezTamayo, J. C.; Ardá, A.; Jiménez-Barbero, J.; Pardo, L.; Rodríguez, R. E.; Arsequell, G.; Valencia, G. Modulation of the Interaction between a Peptide Ligand and a G Protein-Coupled Receptor by Halogen Atoms. ACS Med. Chem. Lett. 2015, 6, $872-876$.

(77) Celis-Barros, C.; Saavedra-Rivas, L.; Salgado, J. C.; Cassels, B. K.; Zapata-Torres, G. Molecular dynamics simulation of halogen bonding mimics experimental data for cathepsin L inhibition. J. Comput. Aided Mol. Des. 2015, 29, 37-46.

(78) González-Vera, J. A.; Medina, R. A.; Martín-Fontecha, M.; Gonzalez, A.; de La Fuente, T.; Vázquez-Villa, H.; García-Cárceles, J.; Botta, J.; McCormick, P. J.; Benhamú, B.; Pardo, L.; López-Rodríguez, M. A new serotonin 5-HT6 receptor antagonist with procognitive activity-Importance of a halogen bond interaction to stabilize the binding. Sci. Rep. 2017, \%, 41293.

(79) Jiang, S.; Zhang, L.; Cui, D.; Yao, Z.; Gao, B.; Lin, J.; Wei, D. The Important Role of Halogen Bond in Substrate Selectivity of Enzymatic Catalysis. Sci. Rep. 2016, 6, 34750 .

(80) Gautieri, A.; Milani, A.; Pizzi, A.; Rigoldi, F.; Redaelli, A.; Metrangolo, P. Molecular dynamics investigation of halogenated amyloidogenic peptides. J. Mol. Model. 2019, 25,124 .

(81) Lameira, J.; Bonatto, V.; Cianni, L.; dos Reis Rocho, F.; Leitão, A.; Montanari, C. A. Predicting the affinity of halogenated reversible covalent inhibitors through relative binding free energy. Phys. Chem. Chem. Phys. 2019, in press.

(82) Bayly, C. I.; Cieplak, P.; Cornell, W. D.; Kollman, P. A. A well-behaved electrostatic potential based method using charge restraints for deriving atomic charges: the RESP model. J. Chem. Phys. 1993, 97, 10269-10280. 
(83) Rendine, S.; Pieraccini, S.; Forni, A.; Sironi, M. Halogen bonding in ligand-receptor systems in the framework of classical force fields. Phys. Chem. Chem. Phys. 2011, 13, $19508-19516$.

(84) Franchini, D.; Dapiaggi, F.; Pieraccini, S.; Forni, A.; Sironi, M. Halogen bonding in the framework of classical force fields: The case of chlorine. Chem. Phys. Lett. 2018, 712, 89-94.

(85) Kolář, M.; Hobza, P. On extension of the current biomolecular empirical force field for the description of halogen bonds. J. Chem. Theory Comput. 2012, 8, 1325-1333.

(86) Kolář, M.; Hobza, P.; Bronowska, A. K. Plugging the explicit $\sigma$-holes in molecular docking. Chem. Commun. 2013, 49, 981-983.

(87) Titov, O. I.; Shulga, D. A.; Palyulin, V. A. Quadrupole Correction: From Molecular Electrostatic Potential to Free Energies of Halogen Bonding. J. Chem. Theory Comput. 2019, 15, 1159-1167.

(88) Jorgensen, W. L.; Schyman, P. Treatment of halogen bonding in the OPLS-AA force field: application to potent anti-HIV agents. J. Chem. Theory Comput. 2012, 8, 38953901.

(89) Harder, E. et al. OPLS3: a force field providing broad coverage of drug-like small molecules and proteins. J. Chem. Theory Comput. 2015, 12, 281-296.

(90) Roos, K.; Wu, C.; Damm, W.; Reboul, M.; Stevenson, J. M.; Lu, C.; Dahlgren, M. K.; Mondal, S.; Chen, W.; Wang, L.; Abel, R.; Friesner, R. A.; Harder, E. D. OPLS3e: Extending force field coverage for drug-like small molecules. J. Chem. Theory Comput. 2019, 15, 1863-1874.

(91) Gutiérrez, I. S.; Lin, F.-Y.; Vanommeslaeghe, K.; Lemkul, J. A.; Armacost, K. A.; Brooks, C. L.; MacKerell, A. D. Parametrization of halogen bonds in the CHARMM 
general force field: Improved treatment of ligand-protein interactions. Bioorg. Med. Chem. 2016, 24, 4812-4825.

(92) Zhou, Y.; Wang, Y.; Li, P.; Huang, X.-P.; Qi, X.; Du, Y.; Huang, N. Exploring Halogen Bonds in 5-Hydroxytryptamine 2B Receptor-Ligand Interactions. ACS Med. Chem. Lett. 2018, 9, 1019-1024.

(93) Nunes, R.; Vila-Viçosa, D.; Costa, P. J. Tackling Halogenated Species with PBSA: Effect of Emulating the $\sigma$-Hole. J. Chem. Theory Comput. 2019, 15, 4241-4251.

(94) Luchi, A. M.; Angelina, E. L.; Andujar, S. A.; Enriz, R. D.; Peruchena, N. M. Halogen bonding in biological context: a computational study of D2 dopamine receptor. $J$. Phys. Org. Chem. 2016, 29, 645-655.

(95) Rana, N.; Conley, J. M.; Soto-Velasquez, M.; Leon, F.; Cutler, S. J.; Watts, V. J.; Lill, M. A. Molecular Modeling Evaluation of the Enantiomers of a Novel Adenylyl Cyclase 2 Inhibitor. J. Chem. Inf. Model. 2017, 57, 322-334.

(96) Costa, P. J.; Nunes, R. In Frontiers in Computational Chemistry: Volume 4; UlHaq, Z., Wilson, A. K., Eds.; Bentham Science Publishers: Sharjah, UAE, 2018; Chapter 4, pp 144-183.

(97) Carter, M.; Rappe, A. K.; Ho, P. S. Scalable anisotropic shape and electrostatic models for biological bromine halogen bonds. 2012, 8, 2461-2473.

(98) Scholfield, M. R.; Ford, M. C.; Vander Zanden, C. M.; Billman, M. M.; Ho, P. S.; Rappé, A. K. Force field model of periodic trends in biomolecular halogen bonds. J. Phys. Chem. B 2015, 119, 9140-9149.

(99) Santos, L. A.; da Cunha, E. F.; Ramalho, T. C. Toward the classical description of halogen bonds: a quantum based generalized empirical potential for fluorine, chlorine, and bromine. J. Phys. Chem. A 2017, 121, 2442-2451. 
(100) Du, L.; Gao, J.; Bi, F.; Wang, L.; Liu, C. A polarizable ellipsoidal force field for halogen bonds. J. Comput. Chem. 2013, 34, 2032-2040.

(101) Mu, X.; Wang, Q.; Wang, L.-P.; Fried, S. D.; Piquemal, J.-P.; Dalby, K. N.; Ren, P. Modeling organochlorine compounds and the $\sigma$-hole effect using a polarizable multipole force field. J. Phys. Chem. B 2014, 118, 6456-6465.

(102) Lin, F.-Y.; MacKerell Jr, A. D. Improved Modeling of Halogenated Ligand-Protein Interactions Using the Drude Polarizable and CHARMM Additive Empirical Force Fields. J. Chem. Inf. Model. 2018, 59, 215-228.

(103) Hariharan, P. C.; Pople, J. A. The influence of polarization functions on molecular orbital hydrogenation energies. Theor. Chim. Acta 1973, 28, 213-222.

(104) Francl, M. M.; Pietro, W. J.; Hehre, W. J.; Binkley, J. S.; Gordon, M. S.; DeFrees, D. J.; Pople, J. A. Self-consistent molecular orbital methods. XXIII. A polarization-type basis set for second-row elements. J. Chem. Phys. 1982, 77, 36543665 .

(105) Rassolov, V. A.; Ratner, M. A.; Pople, J. A.; Redfern, P. C.; Curtiss, L. A. 6-31G* basis set for third-row atoms. J. Comput. Chem. 2001, 22, 976-984.

(106) Glukhovtsev, M. N.; Pross, A.; McGrath, M. P.; Radom, L. Extension of Gaussian-2 (G2) theory to bromine-and iodine-containing molecules: Use of effective core potentials. J. Chem. Phys. 1995, 103, 1878-1885.

(107) Frisch, M. J. et al. Gaussian 09 Revision D.01. Gaussian Inc. Wallingford CT 2009.

(108) The MK atomic radius for iodine is unknown in Gaussian09. Therefore, the default atomic radius for bromine $(2.3 \AA)$ was assigned.

(109) Wang, J.; Wang, W.; Kollman, P. A.; Case, D. A. Automatic atom type and bond 
type perception in molecular mechanical calculations. J. Mol. Graph. Model. 2006, 25, 247-260.

(110) Case, D. A. et al. AMBER 2015. University of California, San Francisco, 2015.

(111) da Silva, A. W. S.; Vranken, W. F. ACPYPE-AnteChamber PYthon Parser interfacE. BMC Res. Notes 2012, 5, 367.

(112) Berendsen, H. J. C.; van der Spoel, D.; van Drunen, R. GROMACS: a message-passing parallel molecular dynamics implementation. Comput. Phys. Commun. 1995, 91, 4356.

(113) Van Der Spoel, D.; Lindahl, E.; Hess, B.; Groenhof, G.; Mark, A. E.; Berendsen, H. J. C. GROMACS: fast, flexible, and free. J. Comput. Chem. 2005, 26, 1701-1718.

(114) Abraham, M. J.; Murtola, T.; Schulz, R.; Páll, S.; Smith, J. C.; Hess, B.; Lindahl, E. GROMACS: high performance molecular simulations through multi-level parallelism from laptops to supercomputers. SoftwareX 2015, 1, 19-25.

(115) Dickson, C. J.; Madej, B. D.; Skjevik, Å. A.; Betz, R. M.; Teigen, K.; Gould, I. R.; Walker, R. C. Lipid14: The Amber Lipid Force Field. J. Chem. Theory Comput. 2014, $10,865-879$.

(116) Jorgensen, W. L.; Chandrasekhar, J.; Madura, J. D.; Impey, R. W.; Klein, M. L. Comparison of simple potential functions for simulating liquid water. J. Chem. Phys. 1983, 79, 926-935.

(117) Bussi, G.; Donadio, D.; Parrinello, M. Canonical sampling through velocity rescaling. J. Chem. Phys. 2007, 126, 014101.

(118) Berendsen, H. J.; Postma, J. v.; van Gunsteren, W. F.; DiNola, A.; Haak, J. R. Molecular dynamics with coupling to an external bath. J. Chem. Phys. 1984, 81, 3684-3690. 
(119) Darden, T.; York, D.; Pedersen, L. Particle mesh Ewald: an $N \cdot \log (N)$ method for Ewald sums in large systems. J. Chem. Phys. 1993, 98, 10089-10092.

(120) Essmann, U.; Perera, L.; Berkowitz, M. L.; Darden, T.; Lee, H.; Pedersen, L. G. A smooth particle mesh Ewald method. J. Chem. Phys. 1995, 103, 8577-8593.

(121) Pall, S.; Hess, B. A flexible algorithm for calculating pair interactions on SIMD architectures. Comput. Phys. Commun. 2013, 184, 2641-2650.

(122) Hess, B. P-LINCS: A Parallel Linear Constraint Solver for Molecular Simulation. J. Chem. Theory Comput. 2008, 4, 116-122.

(123) Hess, B.; Bekker, H.; Berendsen, H. J.; Fraaije, J. G. LINCS: a linear constraint solver for molecular simulations. J. Comput. Chem. 1997, 18, 1463-1472.

(124) Miyamoto, S.; Kollman, P. A. SETTLE: an analytical version of the SHAKE and RATTLE algorithm for rigid water models. J. Comput. Chem. 1992, 13, 952-962.

(125) Silverman, B. W. Density estimation for statistics and data analysis; Chapman and Hall: London, 1986.

(126) Campos, S. R. R.; Baptista, A. M. Conformational analysis in a multidimensional energy landscape: study of an arginylglutamate repeat. J. Phys. Chem. B 2009, 113, 15989-16001.

(127) The PyMOL Molecular Graphics System, Version 2.0 Schrödinger, LLC.

(128) Zhang, Q.; Xu, Z.; Zhu, W. The underestimated halogen bonds forming with protein side chains in drug discovery and design. J. Chem. Inf. Model. 2017, 57, 22-26.

(129) Lu, Y.; Wang, Y.; Zhu, W. Nonbonding interactions of organic halogens in biological systems: implications for drug discovery and biomolecular design. Phys. Chem. Chem. Phys. 2010, 12, 4543-4551. 
(130) Kabsch, W.; Sander, C. Dictionary of protein secondary structure: pattern recognition of hydrogen-bonded and geometrical features. Biopolymers: Original Research on Biomolecules 1983, 22, 2577-2637. 